\title{
A catalogue of white dwarfs in Gaia EDR3
}

\author{
N. P. Gentile Fusillo ${ }^{\circledR},{ }^{1 \star}$ P.-E. Tremblay, ${ }^{2}$ E. Cukanovaite ${ }^{\circledR},{ }^{2}$ A. Vorontseva, ${ }^{3}$ R. Lallement, ${ }^{4}$ \\ M. Hollands ${ }^{\oplus},{ }^{2}$ B. T. Gänsicke ${ }^{\odot},{ }^{2}$ K. B. Burdge ${ }^{\oplus},{ }^{5}$ J. McCleery ${ }^{2}$ and S. Jordan ${ }^{\oplus 6}$ \\ ${ }^{1}$ European Southern Observatory, Karl Schwarzschild Straße 2, D-85748 Garching, Germany \\ ${ }^{2}$ Department of Physics, University of Warwick, Coventry CV4 7AL, UK \\ ${ }^{3}$ Sixfold GmbH, Magirus-Deutz-Straße 16, D-89077 Ulm, Germany \\ ${ }^{4}$ GEPI (Galaxies-Etoiles-Physique-Instrumentation), Observatoire de Paris, 5 Place Jules Janssen, F-92195 Meudon Cedex, France \\ ${ }^{5}$ Division of Physics, Mathematics and Astronomy, California Institute of Technology, Pasadena, CA 91125, USA \\ ${ }^{6}$ Astronomisches Rechen-Institut, Zentrum für Astronomie, Universität Heidelberg, Mönchhofstraße 12-14, D-69120 Heidelberg, Germany
}

Accepted 2021 September 14. Received 2021 September 14; in original form 2021 June 14

\begin{abstract}
We present a catalogue of white dwarf candidates selected from Gaia Early Data Release 3 (EDR3). We applied several selection criteria in absolute magnitude, colour, and Gaia quality flags to remove objects with unreliable measurements while preserving most stars compatible with the white dwarf locus in the Hertzsprung-Russell diagram. We then used a sample of over 30000 spectroscopically confirmed white dwarfs and contaminants from the Sloan Digital Sky Survey (SDSS) to map the distribution of these objects in the Gaia absolute magnitude-colour space. Finally, we adopt the same method presented in our previous work on Gaia Data Release 2 (DR2) to calculate a probability of being a white dwarf $\left(P_{\mathrm{WD}}\right)$ for $\simeq 1.3$ million sources that passed our quality selection. The $P_{\mathrm{WD}}$ values can be used to select a sample of $\simeq 359000$ high-confidence white dwarf candidates. We calculated stellar parameters (effective temperature, surface gravity, and mass) for all these stars by fitting Gaia astrometry and photometry with synthetic pure- $\mathrm{H}$, pure-He, and mixed $\mathrm{H}-\mathrm{He}$ atmospheric models. We estimate an upper limit of 93 per cent for the overall completeness of our catalogue for white dwarfs with $G \leq 20 \mathrm{mag}$ and effective temperature $\left(T_{\text {eff }}\right)>7000 \mathrm{~K}$, at high Galactic latitudes $\left(|b|>20^{\circ}\right.$ ). Alongside the main catalogue we include a reduced proper motion extension containing $\simeq 10200$ white dwarf candidates with unreliable parallax measurements that could, however, be identified on the basis of their proper motion. We also performed a cross-match of our catalogues with SDSS Data Release 16 (DR16) spectroscopy and provide spectral classification based on visual inspection for all resulting matches.
\end{abstract}

Key words: catalogues - surveys - white dwarfs.

\section{INTRODUCTION}

White dwarfs are by far the most common stellar remnants in the Galaxy and over 95 per cent of all stars will end their lives as one of these small fading members (Fontaine, Brassard \& Bergeron 2001). Several unique properties of white dwarfs make them powerful tools with applications in various areas of astronomy: from flux calibration (e.g. Bohlin, Gordon \& Tremblay 2014) to cosmochronology (e.g. Fontaine et al. 2001) and exoplanetary science (e.g. Hollands, Gänsicke \& Koester 2018a). However, the intrinsic low luminosity of white dwarfs has always posed a significant observational challenge and large, well-defined samples of these stars have historically been difficult to assemble.

In 2018 the Gaia Data Release 2 (DR2) led to a true revolution in the field of white dwarf science, with accurate parallax measurements unlocking the possibility to search for these stellar remnants on an unprecedented scale. Jiménez-Esteban et al. (2018) identified $\simeq 73000$ white dwarfs and explored in more details the population within the $100 \mathrm{pc}$ solar neighbourhood, and Gentile Fusillo et al.

^E-mail: ngentile@eso.org
(2019) sampled the entirety of Gaia DR2 identifying a total of $\simeq 260000$ white dwarfs, an eightfold increment compared to the number of objects known before Gaia (Gentile Fusillo et al. 2019). This new, well-defined, and homogeneous sample of white dwarfs gave astronomers an unprecedented opportunity to look at the global properties of these stars, resulting already in a number of important new discoveries.

Tremblay et al. (2019b) identified a 'transversal' sequence in the Hertzsprung-Russell (H-R) diagram of Gaia white dwarfs not aligned with theoretical cooling tracks and not explained by a unique atmospheric composition. Tremblay et al. (2019b) recognized this feature as the first direct observational evidence of a delay in white dwarf cooling due to core crystallization and associated physics such as phase separation and sedimentation, a feature of the H$\mathrm{R}$ diagram that had been predicted over $50 \mathrm{yr}$ before (van Horn 1968). Cheng, Cummings \& Ménard (2019) later demonstrated that about 6 per cent of high-mass white dwarfs $\left(M>1.05 \mathrm{M}_{\odot}\right)$ on this transverse sequence, likely the products of double-degenerate mergers, must experience an extra 8 Gyr cooling delay not explained by core crystallization alone. More recently, Blouin, Daligault \& Saumon (2021) reconciled these results showing that a distillation process during ${ }^{22} \mathrm{Ne}$ phase separation in crystallizing white dwarfs 
could explain both the cooling delay of standard white dwarfs and the extra delay experienced by high-mass double white dwarf mergers (see also Bauer et al. 2020; Camisassa et al. 2021). A number of additional studies have focused on the spectral properties of ultramassive white dwarfs, consolidating the idea that many of these systems are the result of double white dwarf mergers (Hollands et al. 2020; Kawka, Vennes \& Ferrario 2020; Kilic et al. 2021).

In addition to enabling a close look at the $\mathrm{H}-\mathrm{R}$ diagram of white dwarfs, the parallax measurements of Gaia allowed to more precisely estimate white dwarf fundamental parameters and also calculate them independently of spectroscopy. Consequently, in the wake of DR2 a number of studies revisited the stellar parameters of various subsets of white dwarfs, evaluated potential systematic offsets in the data of Gaia and of various additional large-area surveys, and provided a new statistical view on the global properties of white dwarfs (see e.g. Bergeron et al. 2019; Coutu et al. 2019; Ourique et al. 2019; Tremblay et al. 2019a; Chandra et al. 2020). The white dwarf luminosity function was also reexplored with unprecedented level of detail (Torres et al. 2021); and Torres et al. (2019) further investigated the memberships of white dwarfs into the thin disc, thick disc, and halo Galactic populations. Significant progress was also made for largescale identification and characterization of white dwarfs in binaries with main-sequence stars, either in common proper motion pairs (El-Badry, Rix \& Weisz 2018), non-interacting unresolved systems (Inight et al. 2021), or cataclysmic variables (Abril et al. 2020; Pala et al. 2020).

In addition to providing new insight into the global properties of white dwarfs, the huge number of new objects discovered thanks to Gaia opened-up the opportunity to identify some of the most peculiar and rare types of white dwarfs.

For example, Kaiser et al. (2021) and Hollands et al. (2021) discovered five cool $\left(T_{\text {eff }}<5000 \mathrm{~K}\right)$ white dwarfs with trace $\mathrm{Li}$ in their atmospheres. This rare polluting element is extremely difficult to detect in hotter white dwarfs and could be the signpost of accretion of the crust of a planetary object (Hollands et al. 2021). More discoveries related to planetary systems around white dwarfs enabled by Gaia included WD J0914+1914, a peculiar white dwarf in the process of evaporating a Neptune-like exoplanet (Gänsicke et al. 2019), and the 14 newly identified white dwarfs with gaseous debris from rocky planetesimals (Dennihy et al. 2020; Melis et al. 2020; Gentile Fusillo et al. 2021), which brought the number of such systems known from seven to 21 .

However, despite the enormous progress based on the analysis of the Gaia DR2 white dwarf samples, they are not without limitations. We estimated the Gentile Fusillo et al. (2019) catalogue to be mostly complete only out to $\simeq 70 \mathrm{pc}$, but even within the $20 \mathrm{pc}$ solar neighbourhood a handful of historically known white dwarfs did not have reliable Gaia observations. Furthermore the coolest and therefore reddest white dwarfs remained difficult to be systematically identified both because of their low luminosity and because of relatively high contamination from other red sources with spurious Gaia measurements.

The Early Data Release 3 (EDR3) of Gaia relies on 34 months of observations (compared to 22 months for DR2) and represents an improvement on all fronts over DR2, with parallax measurements being now on average 20-30 per cent more accurate and proper motion measurements twice as accurate as in the previous data release (DR). Additionally, EDR3 includes new flags and diagnostic parameters that allow to better assess the data available for each source and make more robust quality cuts (Gaia Collaboration 2021a; Lindegren et al. 2021; Riello et al. 2021).

\section{IDENTIFYING WHITE DWARFS IN GAIA EDR3}

\subsection{EDR3 quality filtering}

The procedure we employed to select white dwarfs in Gaia EDR3 is in many aspects analogous to the one we developed for Gentile Fusillo et al. (2019), but we do not directly rely on any result from our previous work on DR2. As advised in Fabricius et al. (2021) the EDR3 data set should be considered independent of DR2 and, therefore, we carried out our selection entirely anew. We began by retrieving EDR3 photometry and astrometry for $\simeq 128000$ objects with available spectroscopy in the Sloan Digital Sky Survey (SDSS) Data Release 16 (DR16, Ahumada et al. 2019) with $u-$ $g, g-r$ colours consistent with those of white dwarfs and with PARALLAX_OVER_ERROR $>1$. We visually inspected these spectra and reliably identified a total of 25655 white dwarfs that we used to visualize the full extent of the white dwarf locus in the Gaia $\mathrm{H}-\mathrm{R}$ diagram (Fig. 1). We then implemented a broad cut that defines the area in $\mathrm{H}-\mathrm{R}$ space within which all white dwarfs with reliable EDR3 measurements are expected to be found (equation 1), and limits the number of objects to which all subsequent steps in our selection are applied:

$G_{\mathrm{abs}}>6+5 \times\left(G_{\mathrm{BP}}-G_{\mathrm{RP}}\right)$,

AND PARALLAX_OVER_ERROR $>1$.

It is important to notice that the white dwarf locus defined in this way can only be considered fully inclusive for single white dwarfs, double white dwarf binaries, and white dwarfs with low-luminosity companions that do not significantly contribute to the Gaia colour. For the rest of the paper all mentions of white dwarfs refer only to this type of systems. Some white dwarfs with unresolved mainsequence companions are also included by our initial selection, but the full parameter space spanned by this type of binaries is considerably larger (see figs 3, 6, 8, and 15 in Inight et al. 2021) and a significant fraction of these systems cannot be identified using only Gaia data. Equations (1) and (2) provide a broad definition of the white dwarf locus, but they include a total of 14422222 sources, a large fraction of which have unreliable photometric and astrometric measurements and need to be filtered out. The quality filtering criteria used in Fabricius et al. (2021, RUWE $<1.4$ and IPD_FRAC_MULTI_PEAK $\leq 2$ and IPD_GOF_HARMONIC_AMPLITUDE $<0.1$ ) only remove 20 per cent of the objects in this sample, but, at the same time, exclude $\simeq 11$ per cent of SDSS spectroscopically confirmed white dwarfs with $G<20$ and, therefore, on their own are inadequate for our final aim. In order to maximize the completeness of our final catalogue we defined a series of quality cuts using a combination of several EDR3 parameters. Using our spectroscopic sample as a reference, this selection aims to remove the vast majority of contaminants sources while preserving all stars that genuinely belong in the white dwarf locus.

However, no unique set of quality criteria can be applied uniformly to the entire sky. Crowded areas remain more challenging even in EDR3 and quality cuts that produce relatively clean samples in low-crowding regions do not produce equally good results in more densely populated parts of the sky. Therefore, stricter selection criteria need to be applied for stars in these locations. In order to efficiently deal with this problem, we split the entire EDR3 sample in bins of $\simeq 50 \operatorname{arcsec}^{2}$, counted the objects within each bin and assigned all Gaia sources a DENSITY parameter defined as the total number of objects in its bin. This value can then be used to define 


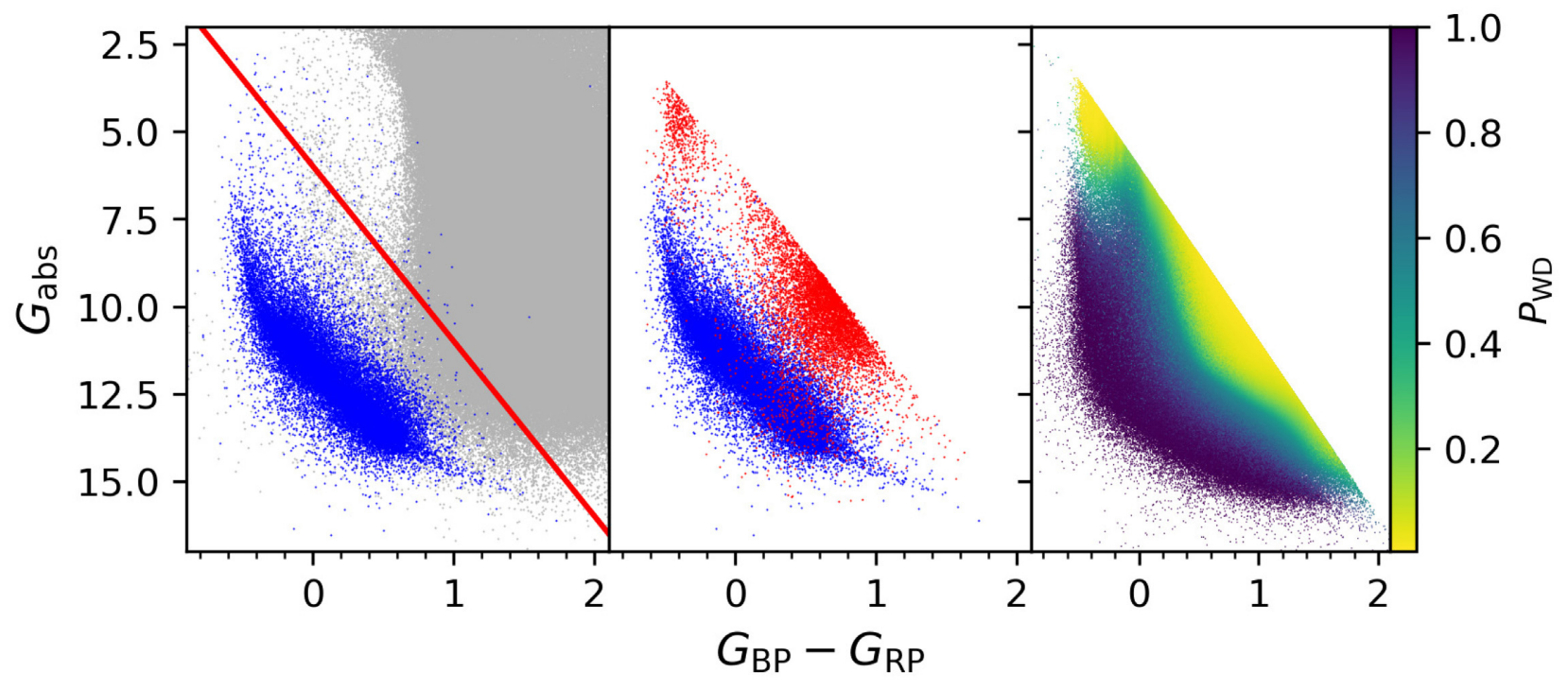

Figure 1. Left-hand panel: Gaia H-R diagram showing a representative sample of 2 million objects (randomly picked using their RANDOM_INDEX) with PARALLAX_OVER_ERROR > 1 (grey points). The blue points represent the SDSS DR16 spectroscopically confirmed white dwarfs used to broadly define the white dwarf locus. The initial cut adopted for our selection is indicated by the red solid lines. Centre panel: distribution of spectroscopically confirmed SDSS white dwarfs (blue) and contaminants (red) included in our final Gaia sample. Right-hand panel: Gaia H-R diagram of all 1280266 objects in our catalogue. The colour scale reflects the $P_{\mathrm{WD}}$ value of each object.

a threshold beyond which stricter selection criteria are required. Additionally we divided the sky in three main areas within which we carried out our quality filtering separately: high galactic latitudes, Galactic plane, and Magellanic Clouds. The Magellanic Clouds area was defined as a $15^{\circ}$ radius around $\alpha=81.28, \delta=-69^{\circ} .78$ (for the Large Magellanic Cloud) plus a $9^{\circ}$ radius around $\alpha=12.80$, $\delta=-73.15$ (for the Small Magellanic Cloud; Gaia Collaboration 2021c).

Sources with DENSITY $<400$ even within the Magellanic Clouds or Galactic plane areas were treated analogously to sources in the High Galactic latitude sample. The final selection criteria adopted are reported in equations (3)-(21).

\section{High Galactic latitudes}

$\left(|b|>25\right.$ OR DENSITY $\left.^{\dagger} \leq 400\right)$,

AND ASTROMETRIC_SIGMA5D_MAX $<1.5$

OR $($ RUWE $\leq 1.1$ AND IPD_GOF_HARMONIC_AMPLITUDE $<1)$,

AND ((PHOT_BP_N_OBS $>$ 2AND PHOT_RP_N_OBS $>2)$

OR PHOT_G_MEAN_MAG $<19)$,

AND (PARALLAX_OVER_ERROR $\geq$ 4OR $\left(\right.$ PM/PM_ERR $\left.\left.{ }^{\dagger}\right)>10\right)$,

AND $\mid$ PHOT_BP_RP_EXCESS_FACTOR_CORRECTED ${ }^{\dagger} \mid<0.6$,

$$
\begin{aligned}
& \text { AND }((\text { ASTROMETRIC_EXCESS_NOISE_SIG }<2 \\
& \text { OR }(\text { ASTROMETRIC_EXCESS_NOISE_SIG } \geq 2 \\
&\text { AND ASTROMETRIC_EXCESS_NOISE }<1.5)) \\
&\text { OR ASTROMETRIC_PARAMS_SOLVED }<32), \\
&\left(|b| \leq 25 \text { AND DENSITY }^{\dagger}>400\right) .
\end{aligned}
$$

\section{Galactic plane}

AND ASTROMETRIC_SIGMA5D_MAX $<1.5$

OR $($ RUWE $\leq 1.1$ AND IPD_GOF_HARMONIC_AMPLITUDE $<1$ ),

AND ((PHOT_BP_N_OBS $>$ 2AND PHOT_RP_N_OBS $>2)$

OR PHOT_G_MEAN_MAG $<19)$,

AND $\left(\right.$ PARALLAX_OVER_ERROR $\geq 4$ OR $\left(\right.$ PM/PM_ERR $\left.\left.{ }^{\dagger}\right)>10\right)$,

AND $\mid$ PHOT_BP_RP_EXCESS_FACTOR_CORRECTED ${ }^{\dagger} \mid<0.6$,

AND ((ASTROMETRIC_EXCESS_NOISE_SIG $<2$

OR (ASTROMETRIC_EXCESS_NOISE_SIG $\geq 2$

AND ASTROMETRIC_EXCESS_NOISE < 1.5))

OR ASTROMETRIC_PARAMS_SOLVED < 32),

AND $\left(\left(\mid\right.\right.$ PHOT_BP_RP_EXCESS_FACTOR_CORRECTED ${ }^{\dagger} \mid<$

$5 \times$ SIGMA_EXCESS_FACTOR $\left.{ }^{\dagger}\right)$

OR (PARALLAX_OVER_ERROR $\geq 4$

AND ASTROMETRIC_SIGMA5D_MAX $\leq 1)$ ),

DENSITY $^{\dagger}>400$,

AND ASTROMETRIC_SIGMA5D_MAX $<1.5$.

Magellanic clouds

AND (PHOT_BP_N_OBS > 2AND PHOT_RP_N_OBS $>$ 2),

AND (PARALLAX_OVER_ERROR $>6$

OR (PARALLAX_OVER_ERROR > 2AND $\left(\right.$ PM/PM_ERR $\left.\left.{ }^{\dagger}\right)>10\right)$ ), 
Table 1. Summary of the white dwarf candidate selection in Gaia EDR3.

\begin{tabular}{lc}
\hline Total number of sources in Gaia EDR3 & 1811709771 \\
Sources in initial colour- $G_{\text {abs }}$ cuts (equations 1-2) & 14422222 \\
No. of objects after quality filtering (equations 3-21) & 1280266 \\
High-confidence candidates $\left(P_{\mathrm{WD}}>0.75\right)$ & 359073 \\
of which with $G \leq 16$ & 2034 \\
of which with $16<G \leq 18$ & 20973 \\
of which with $18<G \leq 20$ & 188784 \\
of which with $G>20$ & 147282 \\
No. of objects in RPM extension (Section 2.3) & 113572 \\
of which with $P_{\mathrm{HWD}}>0.85$ & 10200 \\
\hline
\end{tabular}

\section{AND (ASTROMETRIC_EXCESS_NOISE_SIG $<2$ \\ OR (ASTROMETRIC_EXCESS_NOISE_SIG $\geq 2$ \\ AND ASTROMETRIC_EXCESS_NOISE $<1.5)$ ),}

AND $\mid$ PHOT_BP_RP_EXCESS_FACTOR_CORRECTED ${ }^{\dagger} \mid<$ $5 \times$ SIGMA_EXCESS_FACTOR ${ }^{\dagger}$,

$H_{\mathrm{G}}=$ PHOT_G_MEAN_MAG $+5 \log \mu+5$,

$H_{\mathrm{G}}>10+7 \times\left(G_{\mathrm{BP}}-G_{\mathrm{RP}}\right)$.

The symbol ${ }^{\dagger}$ indicates parameter not provided in the official EDR3 archival distribution, details are provided in the text.

The parameters with the largest impact on our selection are as follows.

ASTROMETRIC_SIGMA5D_MAX, the five-dimensional equivalent to the semimajor axis of the Gaia position error ellipse and is useful for filtering out cases where one of the five parameters, or some linear combination of several parameters, is particularly bad (Lindegren et al. 2018).

PM_OVER_ERR, the ratio of total proper motion to total proper motion error and, although it is not provided in EDR3 archive, it can be calculated from PM, PMRA_ERROR, PMDEC_ERROR.

PHOT_BP_RP_EXCESS_FACTOR_CORRECTED, the PHOT_BP_RP_EXCESS_FACTOR corrected for $G_{\mathrm{BP}}-G_{\mathrm{RP}}$ colour dependence as described in Riello et al. (2021). It is not provided in the EDR3 archive and needs to be calculated following the recipe in Riello et al. (2021), PYTHON code for the calculation is available on public repository. ${ }^{1}$ This parameter can be used to filter out sources with inconsistent $G, G_{\mathrm{BP}}$, and $G_{\mathrm{RP}}$ photometry, which are particularly prominent in crowded regions. In our selection we make cuts in PHOT_BP_RP_EXCESS_FACTOR_CORRECTED with respect to SIGMA_EXCESS_FACTOR that is defined as 'the $1 \sigma$ scatter for a sample of well-behaved isolated stellar sources with good quality Gaia photometry' (see section 9.4 in Riello et al. 2021 for full details).

The combined result of our quality filtering for the three sky areas is a sample of 1280266 objects (Table 1) that represents a compromise between removing the majority of sources with nonoptimal Gaia measurements and preserving all the stars in white dwarf locus.

\subsection{Probability of being a white dwarf: $P_{\mathrm{WD}}$}

Even after applying all the quality filtering described the in previous section, when looking at our sample of 1280266 objects, white

\footnotetext{
${ }^{1}$ https://github.com/agabrown/gaiaedr3-flux-excess-correction
}

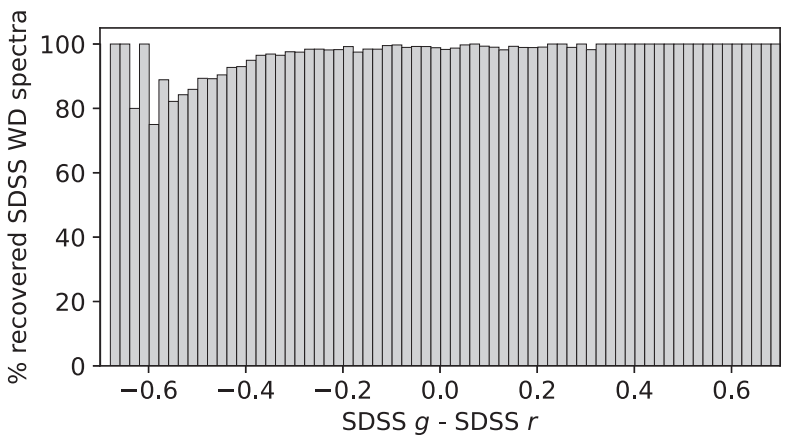

Figure 2. Percentage of SDSS white dwarf spectra identified in our Gaia EDR 3 catalogue as a function of SDSS $g-r$ colour. The $\simeq 20$ per cent drop at $g-r<-0.4$ is likely caused by erroneous inclusion of subdwarfs in the spectroscopic sample.

dwarfs do not immediately stand out as a sequence clearly distinct from the rest of the sources in the sample. Consequently any attempt to select white dwarfs with simple cuts in the $\mathrm{H}-\mathrm{R}$ diagram would result in incomplete and/or contaminated sample.

To answer this problem, we adopted the same procedure described in Gentile Fusillo et al. (2019), i.e. we rely on our sample of spectroscopically confirmed SDSS white dwarfs and contaminants to calculate probabilities of being a white dwarf $\left(P_{\mathrm{WD}}\right)$ for all objects in our Gaia EDR3 sample. We used a total of 22998 spectroscopically confirmed single white dwarfs and 7124 contaminant objects to map their distribution in $\mathrm{H}-\mathrm{R}\left(G_{\mathrm{BP}}-G_{\mathrm{RP}}, G_{\mathrm{abs}}\right)$ space (Fig. 1). In order to create a smooth map covering the entire space of interest, every object was treated as a 2D Gaussian, the width of which reflects the $G_{\mathrm{BP}}-G_{\mathrm{RP}}$ and $G_{\mathrm{abs}}$ uncertainties of the object. For objects with good quality spectra [signal-to-noise-ratio $(\mathrm{S} / \mathrm{N})>10$ ] these Gaussians were normalized so that their volume equals unity, while to reflect the more uncertain classification of objects with low $\mathrm{S} / \mathrm{N}$ spectra $(\mathrm{S} / \mathrm{N}<10)$ we used a normalization value of 0.5 . This results in two continuous smeared-out density maps one for white dwarfs and one for contaminants. A probability map is then created as the ratio of the white dwarf density map to the sum of both density maps. Regions outside our H-R cut (equation 2) where given a fixed probability value of zero. This map can then be used to calculate the $P_{\mathrm{WD}}$ of any Gaia object by integrating the product of its Gaussian distribution in $\mathrm{H}-\mathrm{R}$ space with the underlying probability map.

Our $P_{\mathrm{WD}}$ values allow users to select subsamples of stars flexibly compromising between the desired completeness and acceptable levels of potential contamination.

As a generic guideline selecting objects with $P_{\mathrm{WD}}>0.75$ recovers $\simeq 359000$ high-confidence white dwarf candidates, 25632 of which have SDSS spectroscopy. $\simeq 91$ per cent of these spectroscopic sources are confirmed white dwarfs, $\simeq 1$ per cent are contaminant objects, $\simeq 3$ per cent are white dwarf-main-sequence binaries or cataclysmic variables, and the rest have unreliable classification. When comparing with confirmed SDSS spectroscopic white dwarfs we also find no significant colour bias in this selection (Fig. 2). Cleaner, but less complete, white dwarf subsets can be obtained with higher $P_{\mathrm{WD}}$ thresholds and by imposing additional cuts in Gaia quality parameters stricter than those already adopted in our selection.

\subsection{The reduced proper motion extension}

The location in the H-R diagram of all Gaia sources with PARALLAX_OVER_ERROR $\leq 1$ was considered too unreliable to be used 


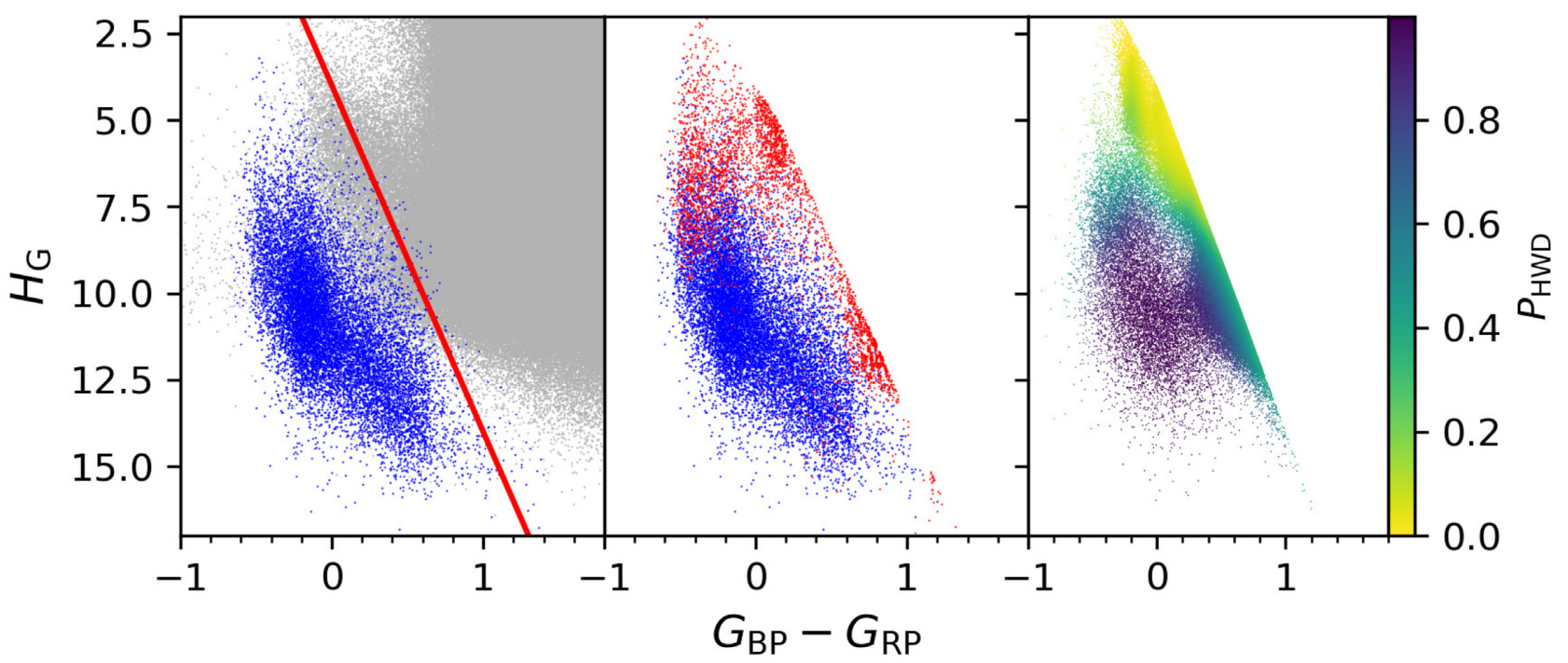

Figure 3. Same as Fig. 1, but showing the distribution of SDSS spectroscopic sources in reduced proper motion (RPM)-colour space and the $P_{\text {HWD }}$ distribution for sources in the RPM extension.

to identify potential white dwarf candidates. However, a significant fraction of these rejected objects have reliable proper motion measurements. Indeed compared to DR2, proper motion measurements in EDR3 are, on average, twice as precise while parallax measurements improved only by 20-30 per cent. In the absence of reliable parallax estimates, reduced proper motion (RPM) can be used as a proxy for distance and can be employed to distinguish different stellar populations. In particular, before the advent of Gaia, colour-RPM diagrams have historically been used to efficiently select white dwarf candidates (e.g. Jones 1972; Harris et al. 2006; Gentile Fusillo, Gänsicke \& Greiss 2015; Lam et al. 2019). With the aim to fully exploit the potential of Gaia as a resource to identify white dwarfs, we decided to create an extension to our main catalogue that contains white dwarf candidates with unreliable parallax measurements, but that could be identified on the basis of their RPM. Similarly to what is described in Section 2 we calculated RPM defined as

\section{AND PARALLAX_OVER_ERROR $\leq 1$}

for all spectroscopically confirmed white dwarfs and contaminants in our SDSS sample and used these objects to determine the locus occupied by white dwarfs in $H_{\mathrm{G}}-\mathrm{BP} \_$RP space (Fig. 3). We then retrieved all Gaia sources with PARALLAX_OVER_ERROR $\leq 1$ and proceeded to define a set of quality cuts aimed at removing sources with unreliable Gaia measurements while preserving objects compatible with the white dwarf locus:

$$
\begin{aligned}
& \text { AND DENSITY }^{\dagger}<800, \\
& \text { AND ASTROMETRIC_SIGMA5D_MAX }<1.5, \\
& \text { AND PHOT_BP_N_OBS }>3, \\
& \text { AND PHOT_RP_N_OBS }>3, \\
& \text { AND }(\text { PM } / \text { PM_ERR } \\
&
\end{aligned}
$$

AND $\mid$ PHOT_BP_RP_EXCESS_FACTOR_CORRECTED ${ }^{\dagger} \mid<$

$$
3 \times \text { SIGMA_EXCESS_FACTOR }^{\dagger},
$$

$A_{G}=0.835 A_{V}$,

$A_{G_{\mathrm{BP}}}=1.139 A_{V}$

This selection results in a sample of 113572 objects (Table 1). Analogously to what is described in Section 2.2 we created a probability map using SDSS spectroscopic white dwarfs, though in this instance using colour-RPM space instead of colour- $G_{\text {abs }}$ space. We then used this map to calculate RPM-based probabilities of being a white dwarf $\left(P_{\mathrm{HWD}}\right)$ for all objects in our RPM extension. Because they are selected among objects with unreliable parallax measurements, all sources in the RPM extension have relatively poor Gaia parameters and are often very faint $(G>20.5)$. Furthermore RPM-based probability maps cannot fully distinguish white dwarfs from hot subdwarfs resulting in some contamination from this type of stars even for relatively high values of $P_{\mathrm{HWD}}$. We therefore suggest the use of the RPM extension only for users interested in the faintest white dwarfs approaching the limit of Gaia detection and recommend selecting objects with $P_{\mathrm{HWD}}>0.85$. We estimate that a total of $\simeq 10200$ genuine white dwarfs are included in the RPM extension. Because of the poorer quality of the Gaia parameters for the objects in the RPM extension, in contrast with the main catalogue, we do not provide extinction estimates (see Section 3.1), stellar parameters (see Section 4), and EXCESS_FLUX_ERROR values (see Section 6) for the white dwarf candidates in this sample.

\section{THE WHITE DWARF CATALOGUE}

A full version of the main Gaia EDR3 catalogue of white dwarf candidates and the RPM extension presented in the previous sections can be downloaded from https://warwick.ac.uk/fac/sci/physics/rese arch/astro/research/catalogues/gaiaedr3_wd_main.fits.gz

https://warwick.ac.uk//fac/sci/physics/research/astro/research/cat alogues/gaiaedr3_wd_rpm_ext.fits.gzand will also be made available via the VizieR catalogue access tool.

All stars in our catalogue are given a name according to the convention presented in Gentile Fusillo et al. (2019), i.e. WD JHHMMSS.SS \pm DDMMSS.SS defined as the white dwarf coordinates in IRCS, at equinox 2000, and epoch 2000. Objects that were 
included in our DR2 catalogue, and already had WDJ names, have not been renamed (even though updated proper motions may have altered their epoch 2000 projected coordinates) and kept their denomination from Gentile Fusillo et al. (2019). The full catalogue format contains all the columns available in the main Gaia EDR3 distribution plus a number of additional ones specific to this work (e.g. $P_{\mathrm{WD}}$, see Table 2). We also include photometric and spectroscopic information from the SDSS (see Section 5) and data from external work on Gaia EDR3. For example, Bailer-Jones et al. (2021) estimated distances for 1.47 billion objects in Gaia EDR3 using a probabilistic approach based on a prior constructed from a three-dimensional Galactic model that included interstellar extinction and the non-uniform magnitude limit of Gaia. Though these distance estimates are not used in the analysis presented in our paper, they represent a valuable added resource for users of our catalogue. We therefore matched all stars in our sample with the Bailer-Jones et al. (2021) catalogue using the unique Gaia EDR3 SOURCE_ID and provide these distance estimates as additional columns.

\subsection{D extinction}

Extinctions are estimated for each white dwarf in the main catalogue by integrating within new, local 3D maps of differential extinction $(d \leq 2.9 \mathrm{kpc})$. The differential extinction at a given point $\mathrm{P}$, i.e. the extinction per unit distance along a line-of-sight crossing $\mathrm{P}$, is proportional to the volume density of the Galactic dust, and is computed by inversion of individual extinctions measured for a large number of target stars distributed in direction and distance. Inversions are based on basic principles described in Vergely et al. (2010) and Lallement et al. (2014) and on recent new, hierarchical techniques presented in Capitanio et al. (2017) in the frame of the STructuring by Inversion the Local Interstellar Medium (STILISM) project and more recently in Lallement et al. (2019). Here, we specifically rely on an unpublished map of Galactic interstellar dust that was computed for the EXPLORE project and will be made available at the CDS and online at https://astro.acri-st.fr/gaia_dev/ (Vergely et al., in preparation). The hierarchical inversion is using extinction catalogues from Sanders \& Das (2018) and Queiroz et al. (2020) that are based on major spectroscopic surveys, and, during the inversion, the map presented in Lallement et al. (2019), which is based on photometry from Gaia and the Two Micron All-Sky Survey (2MASS), is used as a prior distribution and as a final solution in regions of space devoid of spectroscopic survey targets. A more detailed description of this type of data combination, for Sanders \& Das (2018) data solely, can be found in Ivanova et al. (2021). This $4 \times 4 \times 0.8 \mathrm{kpc}^{3}$ new map $(X, Y$ axes in the plane and $Z$-axis along Galactic poles) is particularly well suited for nearby stellar sources such as white dwarfs, because spectroscopic surveys are generally targeting brighter, closer stars. We integrated through the new map based on Gaia 3D coordinates and resulting parameters are found in columns $115-118$ of the catalogue. The extinction $A_{V}$ is given for the standard Johnson $V$ filter and first for the distance corresponding to the exact Gaia $1 / \varpi$ value. Then we derive the range of extinction corresponding to $1 \sigma$ of the parallax value. These estimates neglect uncertainties on the 3D distribution itself and the resulting uncertainty on the integration. Such uncertainties are difficult to quantify individually because they depend on several factors, mainly the target density along the line of sight to the white dwarf, and subsequently the distance, and the uncertainties on the individual extinctions that enter the inversion, but they also depend on the minimum size of clouds reached during the inversion (in our case here $10 \mathrm{pc}$ in most areas). A rough estimate of the average relative error on integrated extinctions is 5 per cent.

In a number of cases the target star is located outside the computational volume of the map and only a lower limit on the extinction is estimated, which corresponds to the extinction reached at the boundary of the map in the direction of the target. Fortunately, in most cases the additional extinction outside the mapped volume is very small, since the dust is concentrated at smaller distance from the plane than our $400 \mathrm{pc}$ map limit. In order to quantify the potential additional extinction generated outside this volume, we have used the dust opacity at $353 \mathrm{GHz}$ measured by Planck to estimate an upper limit on the total extinction up to infinity. The conversion was done based on the recent determination from Remy et al. (2018), i.e. $E(B$ $-V)=1.5 \times 10^{4} \tau 353$ and the average relationship $A_{V}=3.1 E(B-$ $V)$. A flag has been included in the catalogue to distinguish among four cases, from flag 0 for targets either within the map or outside the map and with potential additional extinction $\delta A_{V} \leq 0.2 \mathrm{mag}$ (99.4 per cent of objects), to flag 3 for targets outside the map and with potential additional extinction $\delta A_{V} \geq 1 \mathrm{mag}$ ( $\leq 0.03$ per cent of total).

We do not estimate extinction for the proper motion extension, as these objects have no reliable distances. While reddening could be estimated for these objects in an iterative fashion by assuming an absolute magnitude using white dwarf models, we consider that this model-dependent method is outside the scope of this work.

The derivation of 3D extinction for all white dwarf candidates in the main catalogue is a considerable astrophysical improvement over the DR2 catalogue of Gentile Fusillo et al. (2019), where reddening was instead estimated using a linear distance parametrization of maximum line-of-sight extinction from 2D maps (Schlegel, Finkbeiner \& Davis 1998; Schlafly \& Finkbeiner 2011). We find significant changes in extinction for individual objects, largely due to the presence of local interstellar matter fluctuations that were entirely missed from our earlier 2D parametrization. For white dwarf candidates within $100 \mathrm{pc}$ the new median extinction is $A_{V}=0.020$ mag, with a standard deviation of 0.015 mag, illustrating a fairly homogeneous interstellar medium within this volume. In comparison Gentile Fusillo et al. (2019) proposed a similar median of $A_{V}=0.027$ mag for the same volume, but with a significantly larger and likely unrealistic standard deviation of 0.044 mag.

\section{STELLAR PARAMETERS}

We derive atmospheric parameters for high-confidence white dwarf candidates using a very similar technique and models as in Gentile Fusillo et al. (2019). The main differences compared to our DR2 catalogue, as described in this section, are that we employ EDR3 passbands, use reddening derived from 3D extinction maps, and allow for mixed hydrogen/helium $(\mathrm{H} / \mathrm{He})$ compositions in addition to pure-H and pure-He models.

In recent years Gaia DR2 photometry has been shown to be reliable for deriving white dwarf fundamental parameters, e.g. when compared to the Panoramic Survey Telescope and Rapid Response System (Pan-STARRS), SDSS, or Javalambre Photometric Local Universe Survey (J-PLUS; Bergeron et al. 2019; Gentile Fusillo et al. 2019; López-Sanjuan et al. 2019; Tremblay et al. 2019a; McCleery et al. 2020), albeit with moderate systematic offsets compared to spectroscopic results (Maíz Apellániz \& Weiler 2018; GenestBeaulieu \& Bergeron 2019a; Narayan et al. 2019; Tremblay et al. 2019a, 2020; Cukanovaite et al. 2021). Gaia EDR3 photometric calibration has already been studied extensively (Fabricius et al. 2021; Riello et al. 2021) and is equally reliable as that of DR2 (see 
Table 2. Our catalogue of white dwarfs includes all columns available in the Gaia EDR3 archive. Additional columns unique of this catalogue or not available in the main Gaia EDR3 distribution are summarized here. The full catalogue will be made available via the VizieR catalogue access tool.

\begin{tabular}{ll}
\hline Column & \multicolumn{1}{c}{ Heading } \\
\hline 1 & WHITE_DWARF_NAME \\
3 & DR2_SOURCE_ID \\
12 & ZP_CORRECTION \\
13 & $P_{\text {WD }}$ \\
14 & DENSITY \\
78 & PHOT_G_MEAN_FLUX_CORRECTED \\
79 & PHOT_G_MEAN_MAG_CORRECTED \\
80 & PHOT_G_MEAN_MAG_ERROR_CORRECTED \\
99 & PHOT_BP_RP_EXCESS_FACTOR_CORRECTED \\
113 & EXCESS_FLUX_ERROR \\
114 & BRIGHT_NEIGHBOUR \\
115 & AV_MEAN \\
116 & AV_MIN \\
117 & AV_MAX \\
118 & FLAG_EXT
\end{tabular}

\begin{tabular}{|c|c|}
\hline 119 & $T_{\mathrm{EFF}-}(\mathrm{H})$ \\
\hline 120 & $\sigma_{-} T_{\mathrm{EFF}-}(\mathrm{H})$ \\
\hline 121 & $\mathrm{LOG}_{-} g_{-}(\mathrm{H})$ \\
\hline 122 & $\sigma \log _{-} g_{-}(\mathrm{H})$ \\
\hline 123 & $M_{-}(\mathrm{WD}, \mathrm{H})$ \\
\hline 124 & $\sigma M_{-}(\mathrm{WD}, \mathrm{H})$ \\
\hline 125 & $\chi^{2}(\mathrm{H})$ \\
\hline 126 & $T_{\mathrm{EFF}-}(\mathrm{HE})$ \\
\hline 127 & $\sigma_{-} T_{\mathrm{EFF}-}(\mathrm{HE})$ \\
\hline 128 & $\mathrm{LOG}_{-} g_{-}(\mathrm{HE})$ \\
\hline 129 & $\sigma \log _{-} g_{-}(\mathrm{HE})$ \\
\hline 130 & $M_{-}(\mathrm{WD}, \mathrm{HE})$ \\
\hline 131 & $\sigma M_{-}(\mathrm{WD}, \mathrm{HE})$ \\
\hline 132 & $\chi^{2}-(\mathrm{HE})$ \\
\hline 133 & $T_{\text {EFF-(MIXED) }}$ \\
\hline 134 & $\sigma_{-} T_{\mathrm{EFF}-(\mathrm{MIXED})}$ \\
\hline 135 & LOG _ $g_{-}($MIXED) \\
\hline 136 & $\sigma_{-} \log _{-} g_{\text {_(MIXED) }}$ \\
\hline 137 & $M_{-}(\mathrm{WD}, \mathrm{MIXED})$ \\
\hline 138 & $\sigma M_{-}(\mathrm{WD}, \mathrm{MIXED})$ \\
\hline 139 & $\chi^{2}$-(MIXED) \\
\hline 140 & R_MED_GEO \\
\hline 141 & R_LO_GEO \\
\hline 142 & R_HI_GEO \\
\hline 143 & R_MED_PHOTPGEO \\
\hline 144 & R_LO_PHOTOGEO \\
\hline 145 & R_HI_PHOTOGEO \\
\hline 146 & FIDELITY_V1 \\
\hline 147 & SDSS_NAME \\
\hline 148 & SDSS_CLEAN \\
\hline 149 & $u$ MAG \\
\hline 150 & $u$ MAG_ERR \\
\hline 151 & $g \mathrm{MAG}$ \\
\hline 152 & $g$ MAG_ERR \\
\hline
\end{tabular}

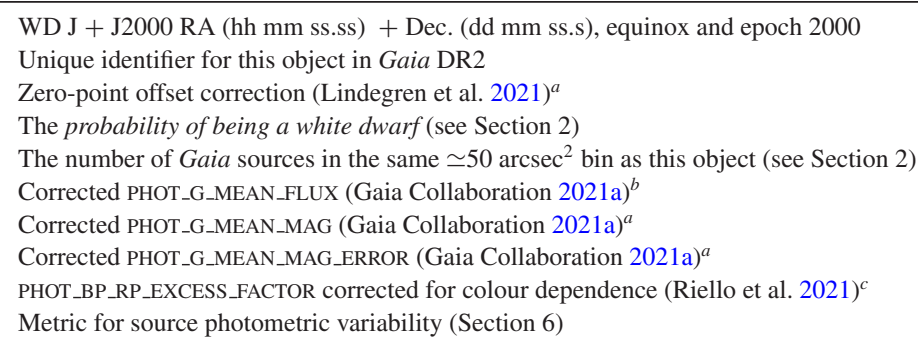

WD J + J2000 RA (hh mm ss.ss) + Dec. (dd mm ss.s), equinox and epoch 2000

Unique identifier for this object in Gaia DR2

Zero-point offset correction (Lindegren et al. 2021) ${ }^{a}$

The probability of being a white dwarf (see Section 2)

The number of Gaia sources in the same $\simeq 50 \operatorname{arcsec}^{2}$ bin as this object (see Section 2)

Corrected PHOT_G_MEAN_FLUX (Gaia Collaboration 2021a) ${ }^{b}$

Corrected PHOT_G_MEAN_MAG (Gaia Collaboration 2021a) $)^{a}$

Corrected PHOT_G_MEAN_MAG_ERROR (Gaia Collaboration 2021a) ${ }^{a}$

PHOT_BP_RP_EXCESS_FACTOR corrected for colour dependence (Riello et al. 2021)

Metric for source photometric variability (Section 6)

If 1 it indicates the presence of a source $5 \mathrm{mag}$ brighter than the target in the $G$-band within 5 arcsec

Mean extinction value (mag) derived from 3D reddening maps (Section 3.1)

Extinction value (mag) derived from 3D reddening maps using $-1 \sigma$ EDR3 distance (Section 3.1)

Extinction value (mag) derived from 3D reddening maps using $+1 \sigma$ EDR3 distance (Section 3.1)

0 indicates that the object is located within the $3 \mathrm{D}$ extinction map or that real extinction value could be $<0.2$ mag larger than AV_MAX

1 indicates that the object is located outside the $3 \mathrm{D}$ extinction map and that real extinction value could be between 0.2 and 0.5 mag larger than AV_MAX

2 indicates that the object is located outside the $3 \mathrm{D}$ extinction map and that real extinction value could be between 0.5 and 1.0 mag larger than AV_MAX

3 indicates that the object is located outside the 3D extinction map and that real extinction value could be $>1.0$ mag larger than AV_MAX (Section 3.1)

Effective temperature (K) from fitting the dereddened $G, G_{\mathrm{BP}}$, and $G_{\mathrm{RP}}$ absolute fluxes with pure-H model atmospheres (see Section 4)

Uncertainty on $T_{\text {eff }-}(\mathrm{H})(\mathrm{K})$

Surface gravity $\left(\mathrm{cm} \mathrm{s}^{-2}\right)$ from fitting the dereddened $G, G_{\mathrm{BP}}$, and $G_{\mathrm{RP}}$ absolute fluxes with pure-H model atmospheres (see Section 4)

Uncertainty on $\log _{-} g_{-}(\mathrm{H})\left(\mathrm{cm} \mathrm{s}^{-2}\right)$

Stellar mass $\left(\mathrm{M}_{\odot}\right)$ resulting from the adopted mass-radius relation and best-fitting parameters (see Section 4)

Uncertainty on $M_{-}(\mathrm{WD}, \mathrm{H})\left(\mathrm{M}_{\odot}\right)$

$\chi^{2}$ value of the fit (pure-H)

Effective temperature (K) from fitting the dereddened $G, G_{\mathrm{BP}}$, and $G_{\mathrm{RP}}$ absolute fluxes with pure-He model atmospheres (see Section 4)

Uncertainty on $T_{\text {eff }}(\mathrm{He})(\mathrm{K})$

Surface gravity $\left(\mathrm{cm} \mathrm{s}^{-2}\right)$ from fitting the dereddened $G, G_{\mathrm{BP}}$, and $G_{\mathrm{RP}}$ absolute fluxes with pure-He model atmospheres (see Section 4)

Uncertainty on $\log _{-} g_{-}(\mathrm{He})\left(\mathrm{cm} \mathrm{s}^{-2}\right)$

Stellar mass $\left(\mathrm{M}_{\odot}\right)$ resulting from the adopted mass-radius relation and best-fitting parameters (see Section 4)

Uncertainty on $M_{-}(\mathrm{WD}, \mathrm{HE})\left(\mathrm{M}_{\odot}\right)$

$\chi^{2}$ value of the fit (pure-He)

Effective temperature (K) from fitting the dereddened $G, G_{\mathrm{BP}}$, and $G_{\mathrm{RP}}$ absolute fluxes with mixed

$\mathrm{H}-\mathrm{He}$ model atmospheres (see Section 4)

Uncertainty on $T_{\text {eff }}(\mathrm{K})$

Surface gravity $\left(\mathrm{cm} \mathrm{s}^{-2}\right)$ from fitting the dereddened $G, G_{\mathrm{BP}}$, and $G_{\mathrm{RP}}$ absolute fluxes with mixed $\mathrm{H}-\mathrm{He}$ model atmospheres (see Section 4)

Uncertainty on $\log g\left(\mathrm{~cm} \mathrm{~s}^{-2}\right)$

Stellar mass $\left(\mathrm{M}_{\odot}\right)$ resulting from the adopted mass-radius relation and best-fitting parameters (see Section 4)

Uncertainty on the mass $\left(\mathrm{M}_{\odot}\right)$

$\chi^{2}$ value of the fit (mixed $\mathrm{H}-\mathrm{He}$ )

Median of the geometric distance posterior (pc) (Bailer-Jones et al. 2021)

16th percentile of the geometric distance posterior (pc) (Bailer-Jones et al. 2021)

84th percentile of the geometric distance posterior (pc) (Bailer-Jones et al. 2021)

Median of the photogeometric distance posterior (pc) (Bailer-Jones et al. 2021)

16th percentile of the photogeometric distance posterior (pc) (Bailer-Jones et al. 2021)

84th percentile of the photogeometric distance posterior (pc) (Bailer-Jones et al. 2021)

'Astrometric fidelity' metric from Rybizki et al. (2021)

SDSS object name if available (SDSS $+\mathrm{J} 2000$ coordinates)

If 1 the SDSS photometry for this object is considered clean (see

https://www.sdss.org/dr16/tutorials/flags)

SDSS $u$-band magnitude (mag)

SDSS $u$-band magnitude uncertainty (mag)

SDSS $g$-band magnitude (mag)

SDSS $g$-band magnitude uncertainty (mag) 
Table 2 - continued

\begin{tabular}{|c|c|c|}
\hline ftolumn & Heading & Description \\
\hline 153 & $r \mathrm{MAG}$ & SDSS $r$-band magnitude (mag) \\
\hline 154 & rMAG_ERR & SDSS $r$-band magnitude uncertainty (mag) \\
\hline 155 & $i \mathrm{MAG}$ & SDSS $i$-band magnitude (mag) \\
\hline 156 & $i$ MAG_ERR & SDSS $i$-band magnitude uncertainty (mag) \\
\hline 157 & $z$ MAG & SDSS $z$-band magnitude (mag) \\
\hline 158 & $z$ MAG_ERR & SDSS $z$-band magnitude uncertainty (mag) \\
\hline 159 & SDSS_SEPARATION & $\begin{array}{l}\text { Angular separation between the Gaia source and its associated SDSS object, after coordinate } \\
\text { separation }(\operatorname{arcsec})\end{array}$ \\
\hline 160 & SDSS_SPECTRA & Number of SDSS spectra available for this object \\
\hline
\end{tabular}

${ }^{a}$ https://gitlab.com/icc-ub/public/gaiadr3_zeropoint

${ }^{b}$ https://github.com/agabrown/gaiaedr3-6p-gband-correction

${ }^{c}$ https://github.com/agabrown/gaiaedr3-flux-excess-correction

Section 7.5.2). Possible colour calibration offsets are discussed in Section 7.7.

As in the DR2 catalogue we rely exclusively on Gaia photometry and astrometry to fit stellar parameters. By construction, reliable Gaia data are available for all white dwarf candidates in our catalogue. In contrast, combining Gaia data with any other optical photometric survey would require careful quality control, especially for ground-based photometry with significantly different spatial resolution. In addition, the photometric calibration of surveys like SDSS is not fully understood and still relies on ad hoc corrections partially based on white dwarf spectroscopic parameters (Eisenstein et al. 2006). Finally, the lack of constraints on atmospheric composition for most candidates pose a serious challenge to include ultraviolet (UV) or infrared (IR) photometric surveys such as the Galaxy Evolution Explorer (GALEX) or the Wide-field Infrared Survey Explorer (WISE). While pure-H, pure-He, and selected mixed $\mathrm{H} / \mathrm{He}$ models have been shown to accurately model Gaia fluxes in most cases (Bergeron et al. 2019; Tremblay et al. 2019a; McCleery et al. 2020), this conclusion may not be correct for photometry outside of the optical, which could be more sensitive to the $\mathrm{H} / \mathrm{He}$ ratio or presence of metals.

There is a degeneracy between $T_{\text {eff }}$ and reddening when using optical photometry. We therefore rely on estimated extinction (Section 3.1) as an external constraint. We convert extinction to reddening using the standard model of Schlafly \& Finkbeiner (2011), the same as that used in Gentile Fusillo et al. (2019):

$A_{G_{\mathrm{RP}}}=0.650 A_{V}$,

$G_{\text {abs }}^{i}=-2.5 \log \left(\frac{\int S(\lambda)^{i} F(\lambda) \lambda \mathrm{d} \lambda}{\int S(\lambda)^{i} \lambda \mathrm{d} \lambda} \frac{1}{\left(3.08568 \times 10^{19} \mathrm{~cm}\right)^{2}}\right)+C^{i}$,

$F(\lambda)=4 \pi R^{2} H_{\lambda}\left(T_{\text {eff }}, \log g\right)$.

For sources with six-parameter astrometric solutions, the $G$-band magnitude should be corrected for known systematic offsets (Riello et al. 2021). We applied the correction following the PYTHON recipe provided in Gaia Collaboration (2021a) ${ }^{2}$ and used these corrected $G$-band magnitude values to estimate stellar parameters. We provide the corrected magnitude values in our catalogue in the column PHOT_G_MEAN_MAG_CORRECTED. The parallax values used for our fits were also adjusted for the known zero-point offsets according to the corrections described in Lindegren et al. (2021). ${ }^{3}$ We provide the zero-point corrections used in the column ZP_CORRECTION.
We have employed the Gaia EDR3 quantum efficiency $S(\lambda)$ for the $G, G_{\mathrm{BP}}$, and $G_{\mathrm{RP}}$ passbands (Riello et al. 2021) to calculate synthetic absolute magnitudes for filter index $i$ using the relation

$G^{i}=-2.5 \log \left(f^{i}\right)+C^{i}$,

where $3.08568 \times 10^{19} \mathrm{~cm}$ equates to $10 \mathrm{pc}$, and $C^{i}$ are the zero points derived using alpha_lyr_stis_002.fits ${ }^{4}$ as Vega reference spectrum (Riello et al. 2021). The resulting zero points are $C^{G}$ $=-21.48503, C^{\mathrm{BP}}=-20.96683$, and $C^{\mathrm{RP}}=-22.22089 \mathrm{mag}$, respectively. $F(\lambda)$ is the integrated stellar flux in erg s $\mathrm{s}^{-1} \AA^{-1}$ related to the emergent monochromatic Eddington flux $H_{\lambda}$ from model atmospheres as

$f^{i}=\varpi^{2} F^{i}$,

where $R$ is the white dwarf radius and $\log g$ is the surface gravity in cgs units.

For pure-H and pure-He atmospheres were rely on the same model atmospheres as in Gentile Fusillo et al. (2019). In brief, we use the grid of Tremblay, Bergeron \& Gianninas (2011) with Lyman $\alpha$ opacity of Kowalski \& Saumon (2006) for pure-hydrogen composition. The validity range of the grid is $1500<T_{\text {eff }}(\mathrm{K})$ $<140000$ and $6.5<\log g<9.5$. For pure-helium composition we use the grid of Bergeron et al. (2011) in the range of $3500<T_{\text {eff }}$ (K) $<40000$ and $7.0<\log g<9.0$.

A new addition to our catalogue is the use of mixed $\mathrm{H} / \mathrm{He}$ model atmospheres. In comparison to using pure-He models, a mixed composition of $\mathrm{H} / \mathrm{He}=10^{-5}$ has been shown to result in a stable mass distribution for He-rich atmospheres, in much better agreement with the H-rich atmosphere mass distribution and predictions from stellar evolution (Bergeron et al. 2019; McCleery et al. 2020). Therefore we employ a third grid of model atmospheres with $\mathrm{H} / \mathrm{He}$ $=10^{-5}$ composition based on calculations of Tremblay et al. (2014) and McCleery et al. (2020) for the range $2000<T_{\text {eff }}(\mathrm{K})<40000$ and $7.0<\log g<9.0$.

In all cases, the radius in equation (35) and mass are calculated using evolution sequences from Bédard et al. (2020) for thick $\mathrm{H}$-layers (pure-H) or thin H-layers (mixed, pure-He) and $M>0.46$ $\mathrm{M}_{\odot}$. For lower masses, we use the He-core cooling sequences of Serenelli et al. (2001). We emphasize that this implies the fitting of only two independent stellar parameters, in our case $T_{\text {eff }}$ and $\log g$, with mass and radius being fully determined from the model dependence.

\footnotetext{
${ }^{2}$ https://gitlab.com/icc-ub/public/gaiadr3_zeropoint

${ }^{3}$ https://github.com/agabrown/gaiaedr3-6p-gband-correction
} 


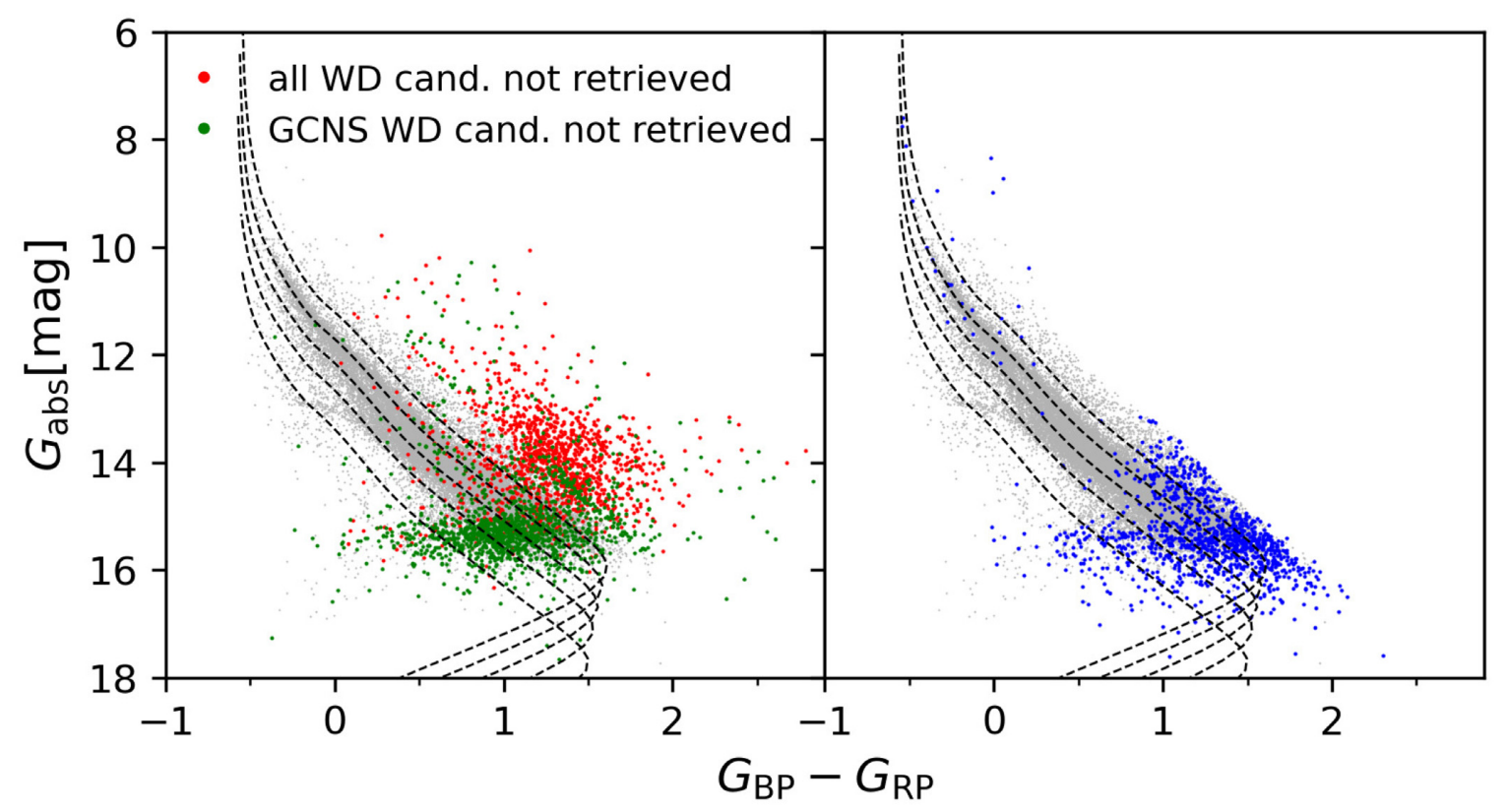

Figure 4. Distribution of objects in common between our catalogue of Gaia EDR3 white dwarf candidates $\left(P_{\mathrm{WD}}>0.75\right)$ and the white dwarfs selected by Gaia Collaboration (2021b, grey points). Objects identified as white dwarf candidates in Gaia Collaboration (2021b) but not recovered by our selection are shown in red, and green if also included in the GCNS (left-hand panel). Vice versa objects identified as nearby white dwarf candidates in this work but not in Gaia Collaboration (2021b) are shown in blue (right-hand panel). The black dashed lines indicate the cooling tracks for H-atmosphere white dwarfs with masses between 0.4 and $1.2 \mathrm{M}_{\odot}$ in steps of $0.2 \mathrm{M}_{\odot}$.

The dereddened observed Gaia flux $f^{i}$ for passband $i$ in units of $\mathrm{erg} \mathrm{cm}^{-2} \mathrm{~s}^{-1}$ is derived from

$P_{\mathrm{WD}}>0.70$,

which is linked to the passband and stellar disc integrated flux $F^{i}$ in $\operatorname{erg~s}^{-1}$ as

AND $\sigma_{T_{\text {eff }}} / T_{\text {eff }}<0.75$.

We employ the same fitting technique as in Gentile Fusillo et al. (2019) based on the non-linear least-squares method of LevenbergMarquardt. The uncertainties on stellar parameters are obtained directly from the covariance matrix. Both the uncertainties and reduced $\chi^{2}$ values are given in our EDR3 catalogue, the latter being useful to flag outliers with colours that deviate from the model grids, such as binaries.

\subsection{Catalogue stellar parameter values}

The full catalogue includes white dwarf candidates that fall outside of the existing model grids, as well as relatively distant objects for which the parallax uncertainty leads to $T_{\text {eff }}$ or $\log g$ error bars that are larger than the full extent of the model grid. Therefore, we adopt the following conditions to have parameters in the catalogue:

AND $\sigma_{\operatorname{logg}}<2.0$

AND $\sigma_{\mathrm{MWD}} / M<1.0$

AND $0.1<M_{\mathrm{WD}} / M<1.4$,

AND $3500<T_{\text {eff }}(\mathrm{K})<140000$,

$3500<T_{\text {eff }}(\mathrm{K})<40000$,
$6600<T_{\text {eff }}(\mathrm{K})<40000$,

with the following additional restrictions for pure-He atmospheres:

EXCESS_FLUX_ERROR

$$
\begin{aligned}
= & \log _{10}(\text { PHOT_G_MEAN_FLUX_ERROR }) \\
& \left.\left.\times \frac{- \text { median }\left(\log _{10}(\text { PHOT_G_MEAN_FLUX_ERROR }\right.}{\text { neighbours }}\right)\right) \\
\text { MAD }\left(\log _{10}(\text { PHOT_G_MEAN_FLUX_ERROR }\right. & \text { neighbours }))
\end{aligned}
$$

and mixed atmospheres pure- $\mathrm{H}$ and mixed parameters are cut-off at low $T_{\text {eff }}$ values compared to the original grid ranges because collision-induced absorption (CIA) opacities are likely incorrect in our current grids of models (Gentile Fusillo et al. 2020). Furthermore, for ultracool white dwarfs past the so-called blue hook in the H-R diagram (Hansen 1998), there is a degeneracy between cool and hot solutions from Gaia data alone. This can be seen from Fig. 4 where pure- $\mathrm{H}$ cooling tracks of mass $M \approx 0.60 \mathrm{M}_{\odot}$ and 2000 $3000 \mathrm{~K}$ cross the cooling tracks of more massive white dwarfs at $3000-6000 \mathrm{~K}$. In our catalogue we always pick the warmer, massive solution, leading to incorrect parameters for ultracool white dwarfs, although only a handful of these objects have so far been identified in the local volume sample (Kilic et al. 2020). As noted in Kilic et al. (2020), these objects may not be ultracool but rather characterized by a peculiar atmospheric composition and faintness in the infrared.

Mass values below $0.2 \mathrm{M}_{\odot}$ and above $1.30 \mathrm{M}_{\odot}$ should be taken with high caution as those parameters were extrapolated outside of the validity range of the available mass-radius relations. Parameters above $40000 \mathrm{~K}$ are extremely sensitive to Gaia colours, zero points and reddening corrections should also be taken with caution without spectroscopic confirmation. Finally, our atmospheric parameters are severely limited for many unresolved binary systems, including double degenerate white dwarfs, although low-mass photometric 
values (overluminous objects) can still be used to identify promising double degenerate candidates (see e.g. Bergeron 2001).

Apart from the limiting cases mentioned above, the catalogued stellar parameters based on state-of-the-art 3D extinction maps are expected to be some of the best available photometric solutions for well-behaved DA, DAH, DAZ (pure-H models), DB, and DC stars (pure-He or mixed models). We refer to table 2 of McCleery et al. (2020) for our proposed choices of solution between pure-H, pure$\mathrm{He}$, or mixed $\mathrm{H} / \mathrm{He}$ as a function of spectral type and temperature. These parameters can be adopted as precise photometric solutions if the spectral type is already know from other sources, e.g. the SDSS-Gaia catalogue in Section 5 or the Montreal White Dwarf Database (Dufour et al. 2017). See Section 7.7 for a comparison of our solutions to spectroscopic parameters.

He-rich atmospheres with temperatures below $\approx 12000 \mathrm{~K}$ have larger uncertainties on their stellar parameters because of the strong effect of trace hydrogen or metal opacities, leading to differences of up to 0.2 dex between the pure-He and mixed solutions (see also Bergeron et al. 2019). We emphasize that while we favour the fixed $\mathrm{H} / \mathrm{He}=10^{-5}$ abundance solution for accurate $T_{\text {eff }}$ and $\log g$ on average (McCleery et al. 2020), it is still not clear if this represents the true $\mathrm{H} / \mathrm{He}$ abundance or if hydrogen is instead a proxy for missing physics in the models, including metal opacities. For cool He-rich atmospheres with detectable metals, carbon or hydrogen (DZ, DZA, $\mathrm{DQ}$, etc.), our parameters should be considered as indicative, as in those cases it is possible to calculate tailored models more appropriate than those used in our catalogue (Hollands et al. 2018a; Blouin \& Dufour 2019; Coutu et al. 2019). Nevertheless, McCleery et al. (2020) have shown that reasonable parameters can be obtained for all He-rich atmospheres, including DQ and DZ stars, using mixed $\mathrm{H} / \mathrm{He}$ models above $7000 \mathrm{~K}$ and pure-He models below that temperature, with the resulting mean mass essentially the same as that of DA white dwarfs in the same temperature range.

\section{THE GAIA-SDSS SPECTROSCOPIC SAMPLE}

We cross-matched our Gaia catalogue of white dwarf candidates with the SDSS DR16 spectroscopic catalogue and retrieved 38740 spectra corresponding to 29254 objects. After visual inspection of the spectra we identified 25176 spectroscopically confirmed white dwarfs (with a total of 33473 spectra). 473 additional white dwarfs with SDSS spectra (with a total of 483 spectra) were found in the RPM extension (Section 2.3). Although this spectroscopic subset only covers $\simeq 7$ per cent of the over 359000 white dwarf candidates in our full catalogue, it still represents the largest sample of spectroscopically confirmed Gaia white dwarfs to date, a record that will most likely be kept until new multi-object spectroscopic facilities [WHT Enhanced Area Velocity Explorer (WEAVE), Dark Energy Spectroscopic Instrument (DESI), 4-metre Multi-Object Spectrograph Telescope (4MOST), and SDSS V] will begin to systematically observe white dwarfs. In our classification of SDSS spectra we adopted 25 classes for white dwarfs: DA, DB, DBA, DAB, DO, DAO, DC, DAZ, DZA, DBZ, DZB, DBAZ, DABZ, DZBA, DZAB, DZ, DQ, hotDQ, DQpec, DAH, DBH, DZH, MWD, PG1159, and WD (Fig. 5; see Sion et al. 1983; Koester 2013 for the definition of these classes). Objects classified as 'MWD' are magnetic white dwarfs where the distortion of spectral features due to the magnetic field is so severe that we were unable to reliably identify the atmospheric composition. Spectra marked as 'WD' have spectra too poor for detailed classification in a subclass, but still broadly recognizable as those of white dwarfs. White dwarfs in binaries and non-white dwarf contaminants were grouped in six

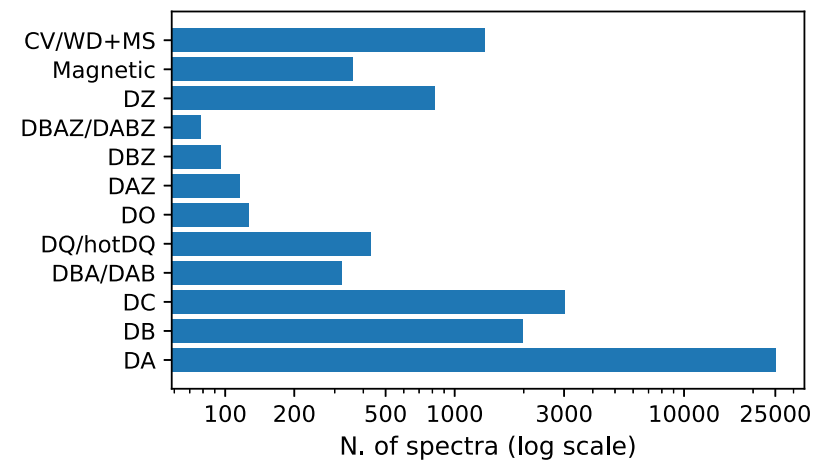

Figure 5. Number of SDSS spectra in each of the main white dwarf spectral classes.

additional spectral classes (CV, DB+MS, DA+MS, DC+MS, STAR, and QSO). Finally, spectra with $\mathrm{S} / \mathrm{N}$ too low for visual classification were simply classed as 'unreliable'. Combined with the Gaia EDR3 data and our stellar parameters (Section 4), this spectroscopic sample represents an ideal tool to further explore the global properties of white dwarfs. The full Gaia-SDSS spectroscopic sample can be downloaded separately from our main catalogue of white dwarfs (Table 3) at the following link: https://warwick.ac.uk//fac/sci/physi cs/research/astro/research/catalogues/gaiaedr3_wd_rpm_ext.fits.gz.

\section{AN INDICATOR OF INTRINSIC VARIABILITY: EXCESS_FLUX_ERROR}

All photometric measurements provided in the Gaia archive are produced by combining the multiple observations the spacecraft obtained for each object. Every individual observation naturally results in slightly different measurements due to a combination of factors including instrumental errors and potentially intrinsic brightness changes in the observed objects. Therefore, the final photometric errors provided in the archive should reflect the overall scatter in the individual Gaia measurements of each objects and so depend on the magnitude of the target, its colour, and the number of observations. Consequently, intrinsically variable stars should acquire additional error due to the increased scatter in brightness measured by Gaia across the different observations. Therefore unusually high photometric errors could be a sign of stellar variability, but to evaluate whether any specific target is a high-error outlier, one has to first establish the typical photometric errors for all objects in the same parameter range (colour, flux, and number of observation) as the target of interest.

For each object in our white dwarf catalogue we retrieved the 500 Gaia sources that clear the selection criteria in equations (4) and (8), and are closest in terms PHOT_G_MEAN_FLUX, number of observations in $G$ (PHOT_G_N_OBS), and colour (BP_RP). This was done by taking the 500 closest objects (neighbours) in terms of the Euclidean distance metric in three-dimensional space. To avoid the dominance of one parameter, they were pre-scaled using a Min-Max approach, i.e. the range of values spanned by each parameter was normalized on a linear scale from 0 to 1 . We find that blue sources in EDR3 have systematically larger relative photometric errors than redder sources prompting the need to limit the neighbour selection to objects of similar colour. The search was also restricted to a specific area of the sky: objects with $|b|<15$ are compared only to other objects within $|b|<15$, and similarly for $|b| \geq 15$. This restriction in position takes care of higher errors due to crowding in regions close to the Galactic plane, while selecting only the closest neighbours 
Table 3. Description of the columns unique to the Gaia EDR3-SDSS spectroscopic catalogue. The full list also includes a number of columns replicated from our main catalogue and so described in Table 2 or in the official EDR3 archive distribution. The full catalogue will be made available online via the VizieR catalogue access tool.

\begin{tabular}{|c|c|c|}
\hline Column no. & Heading & Description \\
\hline 4 & WD_CATALOGUE & $\begin{array}{l}\text { M if the object is included in the main catalogue, } \mathrm{E} \text { if the object is in the RPM extension } \\
\text { (Section 2.3) }\end{array}$ \\
\hline 51 & Plate & Identifier of the plate used in the observation of the spectrum \\
\hline 52 & MJD & Modified Julian Date of the observation of the spectrum \\
\hline 53 & FIBREID & Identifier of the fibre used in the observation of the spectrum \\
\hline 54 & $\mathrm{~S} / \mathrm{N}$ & Signal-to-noise ratio of the spectrum calculated in the range $4500-5500 \AA$ \\
\hline 55 & SPEC_CLASS & Classification of the object based on a visual inspection of the SDSS spectrum \\
\hline
\end{tabular}

Table 4. Summary of the differences in number of selected white dwarf candidates in the Gaia EDR3 catalogue presented here and the DR2 catalogue from Gentile Fusillo et al. (2019).

\begin{tabular}{lc}
\hline White dwarf candidates in EDR3 $\left(P_{\mathrm{WD}}>0.75\right)$ & 359073 \\
White dwarf candidates in DR2 $\left(P_{\mathrm{WD}}>0.75\right)$ & 262480 \\
New candidates in EDR3 & 99151 \\
of which with $G \leq 19$ & 2937 \\
of which completely absent in DR2 & 197 \\
of which with unreliable or incomplete data in DR2 & 98954 \\
DR2 candidates not included in EDR3 catalogue & 9726 \\
of which in 40 pc (according to DR2 parallax) & 22 \\
\hline
\end{tabular}

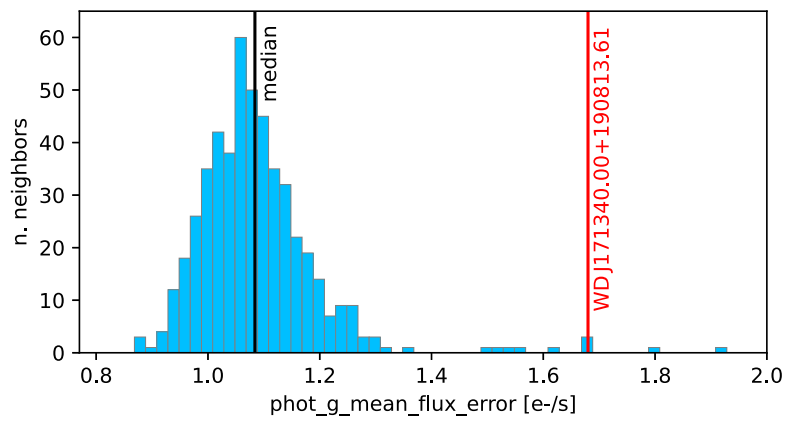

Figure 6. Distribution of PHOT_G_MEAN_MAG_ERROR values of the 500 neighbours of the white dwarf candidate WD J171340.00+190813.61 with EXCESS_FLUX_ERROR $\simeq 8.6$. The black line indicates the median PHOT_G_MEAN_FLUX_ERROR of the neighbours, and the red line indicates the PHOT_G_MEAN_FLUX_ERROR of the white dwarf candidate.

in the aforementioned 3D parameter space ensures the comparison sample only contains objects with similar instrumental error. In order to systematically compare the photometric errors associated with our target to those of the 500 neighbours, we define the quantity EXCESS_FLUX_ERROR as the ratio of the $\log _{10}$ of an object's flux error to the median absolute deviation (MAD) of the $\log _{10}$ of the flux error of its neighbours: We calculated EXCESS_FLUX_ERROR for all objects in our catalogue and include it as a column. Negative values were set to zero. High values of EXCESS_FLUX_ERROR (e.g. >4) indicate that the target object's flux error is significantly higher than that of its neighbours (Fig. 6).

For example, of the 218 cataclysmic variables (CV) in our GaiaSDSS spectroscopic sample, 171 have EXCESS_FLUX_ERROR $>4$, indicating that strongly variable sources can be identified by this parameter. Indeed CVs display some the highest EXCESS_FLUX_ERROR in the catalogue with a median value of $\simeq 13$.

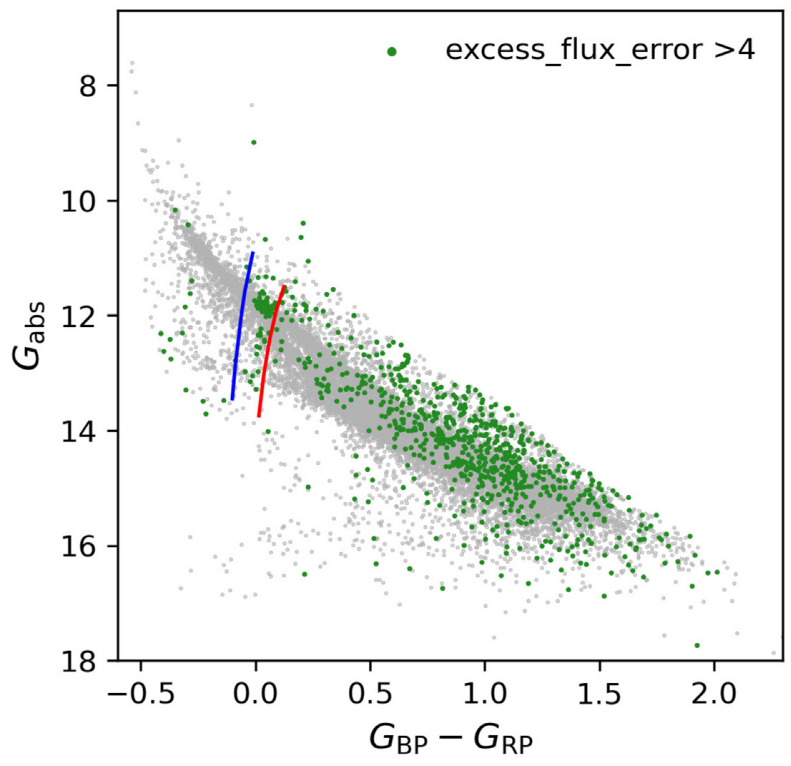

Figure 7. Distribution of all objects in our catalogue with $P_{\mathrm{wd}}>0.75$ and parallax $\geq 10$ (grey points). Sources with EXCESS_FLUX_ERROR $>4$ are plotted as green points. The blue and red edges of the ZZ Ceti empirical instability strip (Gianninas et al.2015) are indicated by the blue and red line, respectively.

As a further test of the potential of the EXCESS_FLUX_ERROR as an indicator of intrinsic variability, we used the empirical ZZ Ceti instability strip defined in Gianninas et al. (2015) and reliable stellar parameters for $\mathrm{H}$-atmosphere white dwarfs $\left(\chi_{(\mathrm{H})}^{2}<1.5\right.$, see Section 4) to select 3295 relatively bright pulsating white dwarf candidates with $G<19$. We find that 5.7 per cent of these stars have EXCESS_FLUX_ERROR $>4$, while outside the empirical instability strip only 1.3 per cent of similarly bright white dwarf candidates have EXCESS_FLUX_ERROR $>4$ (Fig. 7). This simple test indicates that EXCESS_FLUX_ERROR is, in some capacity, sensitive to the brightness variation caused by white dwarf pulsation. However, only a relative small fraction of objects with this level of variability (amplitude 130 per cent; Mukadam et al. 2013) can be reliably identified using this metric.

Guidry et al. (2021) carried out a similar exploration of white dwarf variability in Gaia DR2 by calculating a variability index with a similar scope to our EXCESS_FLUX_ERROR. Though the two metrics are based on different data sets, if they are both capable of identifying genuine variable Gaia sources, there should be a significant overlap in the samples of variable candidates selected using them. We find that about half of the top 1 per cent variable white 
dwarf candidates within 200 pc selected by Guidry et al. (2021) also have EXCESS_FLUX_ERROR $>4$. Additionally all eight new ZZ Ceti that Guidry et al. (2021) identified using their Gaia variability index have EXCESS_FLUX_ERROR $>4.6$.

\section{DISCUSSION}

7.1 Overall completeness: comparison with a SDSS sample of white dwarf candidates

Some intrinsic limits in Gaia observations (e.g. problems with crowding, uneven scanning law, and very broad filters) and the complex selection method behind our final white dwarf sample may cause some genuine white dwarfs to be excluded from our catalogue. In order to quantitatively estimate the combined impact of these factors, we need to compare the new Gaia catalogue of white dwarfs with an independent, sufficiently large, and well-defined sample of stellar remnants. All the spectroscopic samples of white dwarfs currently available, including SDSS, are severely incomplete and biased by the specific observing strategy adopted, and so illsuited for this task. In Gentile Fusillo et al. (2019) we used an independently constructed sample of white dwarf candidates selected on the basis of their colour and RPM as described in Gentile Fusillo et al. (2015). This sample used SDSS photometry and proper motions from the Gaia-Pan-STARRS1 (PS1)-SDSS (GPS1; Tian et al. 2017) catalogue with no additional Gaia-based input. We opt again to use this sample of white dwarf candidates as a comparison group to test the completeness of our new EDR3 white dwarf catalogue. This also allows a direct comparison with the values obtained for our DR2based catalogue. We note that because of the colour restrictions used in the construction of the SDSS comparison sample, it only contains white dwarfs with $T_{\text {eff }}>7000 \mathrm{~K}$, and an additional $\simeq 14000$ are potentially missing because they lacked reliable proper motion measurement in GPS1. We estimated the SDSS comparison sample to contain $\simeq 75$ per cent of all the white dwarfs observed by SDSS, brighter than $g=20.1$ and with $T_{\text {eff }}>7000 \mathrm{~K}$. For completeness, we also point out that the footprint of the SDSS photometry is mostly limited to high Galactic latitudes with $|b| \gtrsim 20^{\circ}$. For a detailed description of the development and characterization of the SDSS comparison sample, see appendix A in Gentile Fusillo et al. (2019).

Analogously to the procedure in Gentile Fusillo et al. (2019), we begin by selecting a subset of 60739 high-confidence dwarf candidates from the SDSS comparison sample. We estimate that this subset only has 7 per cent contamination while still including 97 per cent of all the white dwarfs in the full sample. We then crossmatched the sky position of these objects (correcting for different epoch of observation) with our Gaia EDR3 catalogue of white dwarf candidates and retrieved a total of 52465 stars. Accounting for the estimated level of contamination of the SDSS sample, we can use the number of stars not retrieved in our cross-match to estimate an upper limit in the completeness of the Gaia EDR3 catalogue of 93 per cent. Similarly, we can use the estimated completeness of the SDSS comparison sample and the number of objects retrieved in cross-match to calculate a lower limit in completeness of 67 per cent. In comparison, when performing the same test for our Gaia DR2 catalogue of white dwarfs we estimated a maximum completeness of 85 per cent and a minimum one of 60 per cent. We emphasize that these values can be considered fully descriptive only for white dwarfs with $G \leq 20$ and $T_{\text {eff }}>7000 \mathrm{~K}$, at high Galactic latitudes $\left(|b|>20^{\circ}\right)$.

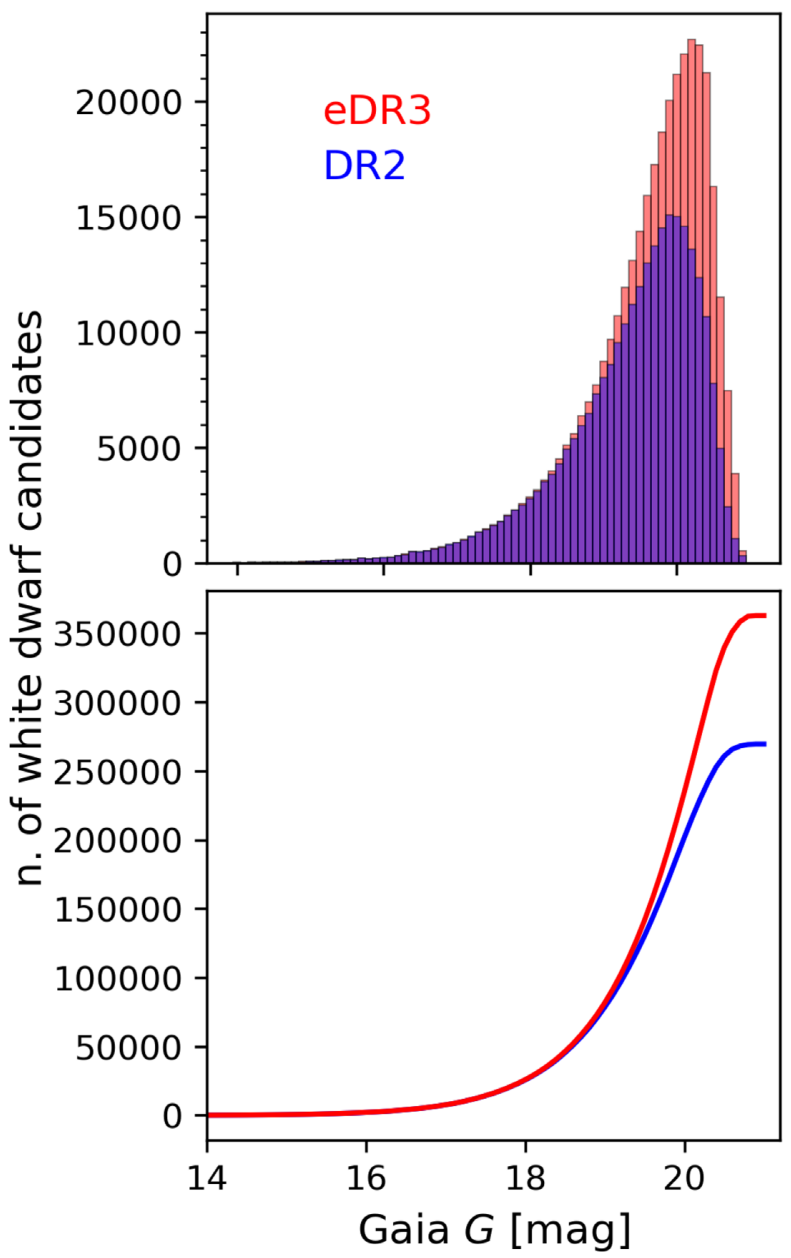

Figure 8. Top panel: number high-confidence white dwarf candidates from Gentile Fusillo et al. $\left(2019, P_{\mathrm{WD}}>0.75\right.$, blue, DR2) and from the catalogue presented here $\left(P_{\mathrm{WD}}>0.75\right.$, red, EDR3) as function of Gaia $G$ magnitude. Bottom panel: same as top panel, but showing the cumulative distribution.

\subsection{New sky density and limiting magnitude: comparison with DR2 catalogue of white dwarf candidates}

In order to assess the improvements brought on by EDR3 compared to DR2, we compared the newly constructed catalogue described here to our Gaia DR2 catalogue of white dwarfs (Gentile Fusillo et al. 2019). We cross-matched the two catalogues by directly comparing the unique Gaia source IDs using the auxiliary table GAIAEDR3.DR2_NEIGHBOURHOOD provided in the Gaia archive. We find that 247505 high-confidence white dwarfs we selected in DR2 (94 per cent) are again identified as white dwarf candidates with $P_{\mathrm{WD}}>0.75$ in our new EDR3 catalogue. In contrast 5249 objects previously identified as reliable white dwarf candidates, while still included in the EDR 3 catalogue, now have $P_{\mathrm{WD}}<0.75$. Additionally 9726 DR2 white dwarf candidates are entirely excluded in the new catalogue. EDR3 photometric and astrometric measurements for these missing sources are either considered unreliable according to our selection criteria (equations 1-21), or place these objects in areas of the H-R diagram not occupied by white dwarfs.

Our EDR3 catalogue also includes $\simeq 99000$ new white dwarf candidates. As illustrated in Fig. 8 the vast majority of the new white dwarfs are fainter than magnitude $G=19$ and for stars brighter than this limit the EDR3 white dwarf catalogue is only 


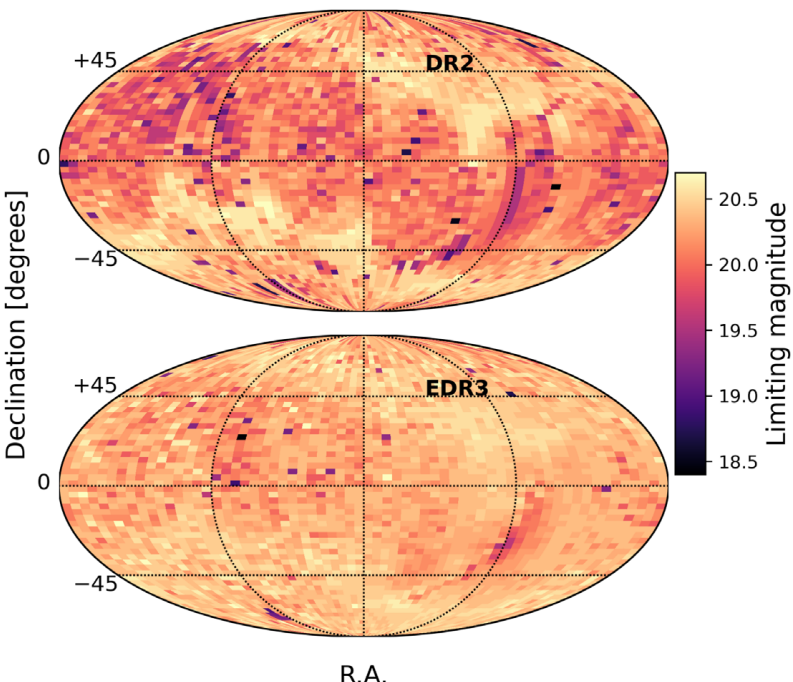

Figure 9. Limiting magnitude for Gaia DR2 white dwarf candidates from Gentile Fusillo et al. (2019) (top panel) and for white dwarf candidates from the new EDR3 catalogue presented here (bottom panel). Both are calculated using $10 \operatorname{deg}^{2}$ bins.

8 per cent larger than its predecessor (Table 4). This is a direct consequence of the improved depth of the EDR3 observations. Most bright white dwarfs already had robust photometric and astrometric data in DR2, but in EDR3 the limiting magnitude for objects with reliable measurements is significantly more uniform across the entire celestial sphere compared to DR2 (Fig. 9). Virtually all parts of the sky are now covered to a magnitude depth of at least $G=20$, and we estimate the sky density of white dwarfs with $G \leq 20$ in EDR3 to range from $4.7 \mathrm{deg}^{-2}$ at $|b|>80$ to $6.1 \mathrm{deg}^{-2}$ at $|b|<10$, with an all-sky average of $\simeq 5.6 \mathrm{deg}^{-2}$.

The increase in number of newly identified white dwarfs is particularly marked in crowded areas of the sky where the improved EDR3 measurements allow us to lift some of the strict limitations we imposed in DR2 for our selection of white dwarfs in these regions (Fig. 10). None the less, even in EDR3, our ability to identify white dwarfs from Gaia data is still reduced in highly crowded areas of the sky compared to less populated areas and, as a result, the completeness of our catalogue drops in the central regions of the Galactic plane (Fig. 11).

\subsection{The 100 pc sample and a comparison with white dwarfs in the Gaia catalogue of nearby stars}

In the Gaia EDR3 release paper (Gaia Collaboration 2021b) the authors include a catalogue of nearby white dwarfs. These stars were identified using a random forest classifier trained on 20000 previously known white dwarfs retrieved from catalogues based on Gaia DR2 astrometry (Jiménez-Esteban et al. 2018; Gentile Fusillo et al. 2019; Torres et al. 2019), with the only initial constraint of parallax $>8$. The classifier tested 1050614 EDR3 sources and assigned to each a probability of being a white dwarf. The authors then considered each object with a probability greater than 0.5 as a valid white dwarf and selected a sample of 32948 white dwarf candidates. Of these objects 21848 also clear all selection criteria to be included in the Gaia Catalogue of Nearby Stars (GCNS; Gaia Collaboration 2021b). From our catalogue we selected a similar sample of highconfidence white dwarf candidates $\left(P_{\mathrm{WD}}>0.75\right.$ and parallax $\left.>8\right)$ and compared it to the selection in Gaia Collaboration (2021b).

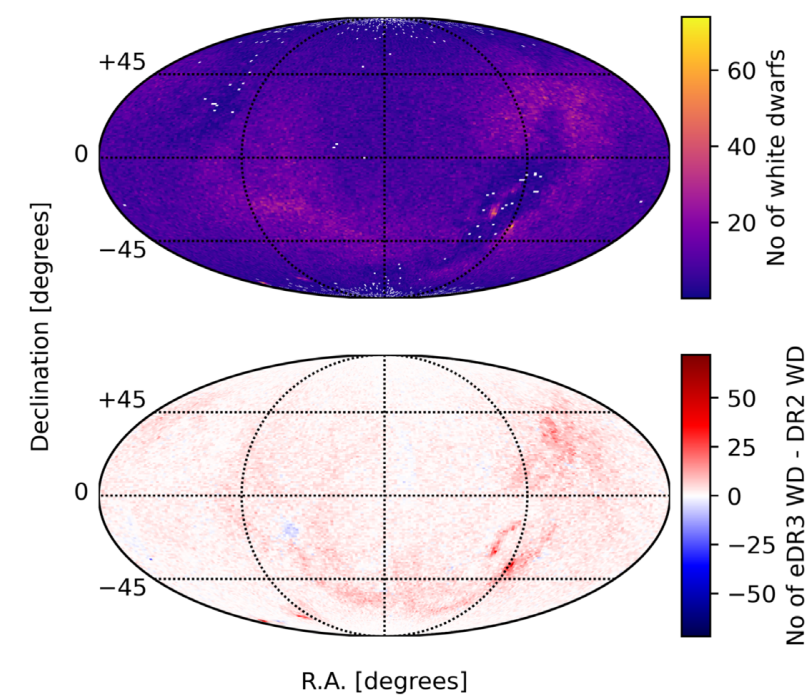

Figure 10 Top panel: Sky density (in $1 \mathrm{deg}^{2}$ bins) of GaiaEDR3 white dwarf candidates with $P_{\mathrm{WD}}>0.75$ from the catalogue presented in this article. Bottom panel: Difference between the number of white dwarf candidatesselected in EDR3 (top panel) and the number of DR2 white dwarf candidates with $P_{\mathrm{WD}}>0.75$ from Gentile Fusillo et al. (2019).

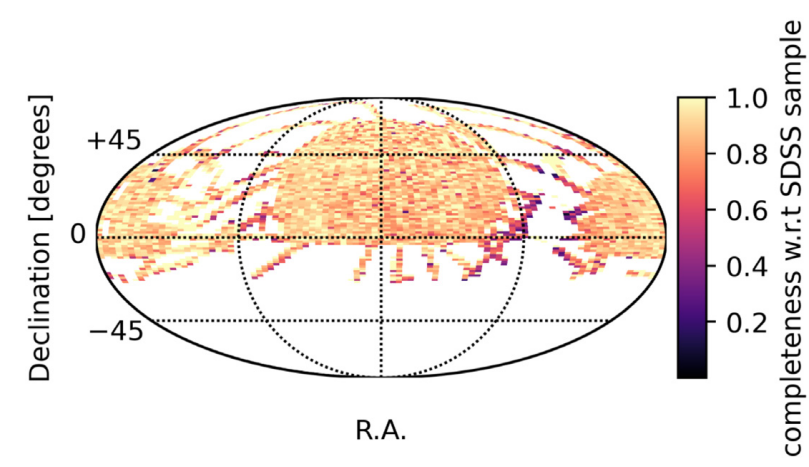

Figure 11. Completeness of our Gaia catalogue of white dwarf candidates with respect to the SDSS comparison sample, as a function of sky position. Each bin represents $5 \mathrm{deg}^{2}$.

We find the two samples in close agreement with 30255 objects in common, but there are also some differences worth of notice. Our catalogue includes 1149 white dwarf candidates not found in Gaia Collaboration (2021b) and similarly Gaia Collaboration (2021b) includes 2693 objects not retrieved by our selection. Even when considering only the 21848 white dwarf candidates in the GCNS, 1419 sources are still not present in our sample (Fig. 4). About half the sources in both unmatched samples (not recovered in our catalogue, but selected in the GCNS and vice versa) are located in dense areas of the Galactic plane where Gaia observations are less reliable. Additionally the objects in both these samples form an horizontal cluster in $\mathrm{H}-\mathrm{R}$ space that does not reflect the predicted locus of white dwarfs, suggesting these objects may not be real white dwarfs (though this effect is more marked for the objects excluded by our catalogue). Fig. 4 also shows two limitations that Gaia Collaboration (2021b) already identified in their white dwarf selection. First, their sample includes a number of objects located above and redward of the white dwarf locus. Most of these stars are not included in the GCNS and Gaia Collaboration (2021b) observed that they may not be true white dwarfs. We suggest that these objects are likely white dwarf 
plus M-dwarf binaries and, though a number of them are also included in our catalogue, most have $P_{\mathrm{WD}}<0.75$ that can distinguish them from well-behaved single white dwarfs. Secondly, $\simeq 30$ bright and relatively hot white dwarfs (some of them historically well known, e.g. Sirius B) are missing from the Gaia Collaboration (2021b) white dwarf sample as these rare objects were not sufficiently represented in the training sample used by the random forest algorithm. From this comparison it is apparent that selecting the coolest and reddest white dwarfs, even within $\simeq 100 \mathrm{pc}$, remains a challenging endeavour, and contamination of the red section of the white dwarf cooling sequence from other sources is something users should be aware of even in EDR3. Consequently it is not possibly to conclusively state weather the Gaia EDR3 100 pc white dwarf sample can be considered complete.

\subsection{Comparison with 'astrometric fidelity' (Rybizki et al. 2021)}

Recently Rybizki et al. (2021) presented a novel approach to quantify the robustness of the astrometric solution for sources in Gaia EDR3. Their method relies on a neural net that uses 14 pertinent Gaia parameters to capture the overall reliability of the Gaia measurements in a single 'astrometric fidelity' parameter.

We retrieved this 'astrometric fidelity' for all sources within our initial selection (equations 1 and 2) and used it as an independent test to verify the robustness of our quality filtering (equations 321). We find that our cuts in ASTROMETRIC_SIGMA5D_MAX, PARALLAX_OVER_ERROR, and PM_OVER_ERR eliminate the vast majority of objects with very low 'astrometric fidelity' $(<0.1)$, confirming the strength of these criteria as discriminators of spurious sources. However our final sample excludes $\simeq 50$ per cent of the 'high-fidelity sources' ('astrometric fidelity' $>0.5$ ) while approximately half of the objects included are 'low-fidelity sources' ('astrometric fidelity' $<0.5$ ). The majority of the 'high-fidelity sources' excluded are located in the densest areas of the Galactic plane or in the Magellanic Clouds and are rejected by our cut on CORRECTED_EXCESS_FACTOR. Additionally most of these rejected objects sit on the boundary of our cut in H-R space (i.e. not on the white dwarf sequence, see Fig. 12).

On the other hand, we also find that $\simeq 40000$ objects in our catalogue with $P_{\mathrm{WD}}>0.75$ are 'low-fidelity sources' (11411 with $G<20$ ). A large fraction of these sources cluster in the upper portion of the white dwarf cooling sequence (Fig. 12) and are most likely moderately hot white dwarfs. Additionally, 1243 SDSS spectroscopically confirmed white dwarfs are among these 'lowfidelity sources' that we identified as reliable high-confidence white dwarf candidates.

In conclusion, the comparison reveals a marked discrepancy between our quality selection and a selection done relying exclusively on the 'astrometric fidelity' computed by Rybizki et al. (2021). This is not surprising as in the current version of their algorithm Rybizki et al. (2021) do not use PHOT_BP_RP_EXCESS_FACTOR as discriminating parameter and their training sample is constructed from sources with a match in the 2MASS and so it is not well suited for faint blue sources like white dwarfs. None the less, the 'astrometric fidelity' could still be useful to a number of users and we decided to include it in our catalogue under the column FIDELITY_V1.

\subsection{Volume complete samples}

The local volume complete sample of white dwarfs, with the Sun at its centre, has historically increased from a radius of $13 \mathrm{pc}$ (Holberg, Oswalt \& Sion 2002) to 20-40 pc (Giammichele, Bergeron \& Dufour 2012; Sion et al. 2014; Limoges, Bergeron \& Lépine 2015), and

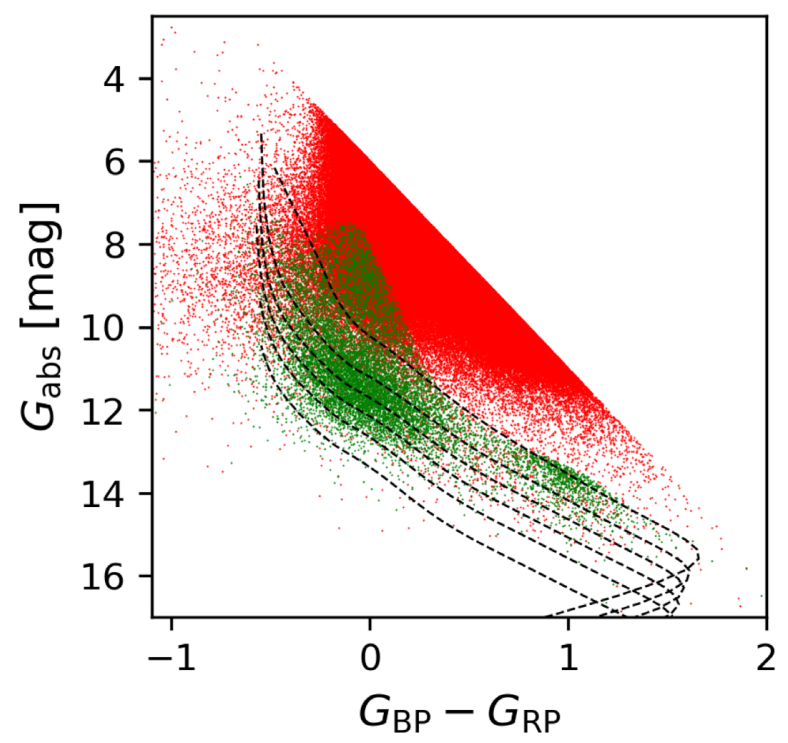

Figure 12. Distribution of 'high-fidelity sources' from Rybizki et al. (2021, 'astrometric fidelity' $>0.5$ ) brighter than $G=20$ excluded from our catalogue (red points), and of 'low-fidelity sources' ('astrometric fidelity' $<0.5$ ) that are instead included with $P_{\mathrm{WD}}>0.75$ (green points). The black dashed lines indicate the cooling tracks for $\mathrm{H}$-atmosphere white dwarfs with masses between 0.4 and $1.2 \mathrm{M}_{\odot}$ in steps of $0.2 \mathrm{M}_{\odot}$.

finally to $\approx 15000$ white dwarf candidates within 100 pc from Gaia DR2 and EDR3 (Jiménez-Esteban et al. 2018; Gentile Fusillo et al. 2019; Gaia Collaboration 2021b). However the vast majority of white dwarf candidates in that Gaia identified sample lack follow-up spectroscopy. Unbiased spectroscopic samples are critically important as they are benchmarks to characterize local space density, stellar formation history, binary evolution or white dwarf spectral evolution, and crystallization. Currently the largest volume complete samples with near-complete ( $>98$ per cent) spectroscopic confirmation are the overlapping $20 \mathrm{pc}$ (Hollands et al. 2018b) and northern $40 \mathrm{pc}$ volumes (McCleery et al. 2020) as identified from Gaia DR2. Work is also progressing towards spectroscopic follow-up of the southern $40 \mathrm{pc}$ sample (in preparation) and $100 \mathrm{pc}$ SDSS footprint (Kilic et al. 2020).

In the following subsections, we describe how our new EDR3 white dwarf catalogue has changed the completeness and properties of the existing 20 and 40 pc volume samples as described specifically by our earlier publications (Hollands et al. 2018b; Gentile Fusillo et al. 2019; McCleery et al. 2020).

\subsubsection{0 pc volume sample}

Compared to both Hollands et al. (2018b) and Gentile Fusillo et al. (2019), the 20 pc volume sample has five new sources in the EDR3 catalogue. Four of them are well-known white dwarfs with missing or incomplete complete data in DR2 (40 Eri B, Ross 627, EGGR 290, and Wolf 489). Another known white dwarf (WD 0728+642) moved from just outside $20 \mathrm{pc}$ to just inside the volume, although it is still within $1 \sigma$ of the boundary. No confirmed 20 pc DR2 white dwarf is missing in our new catalogue or has moved out of the sample. However, four confirmed $20 \mathrm{pc}$ white dwarfs (Procyon B, WD 0208-510, WD 0727+482A, and WD 0727+482B) are missing or have incomplete data in both DR2 and EDR3.

Using the EDR3 catalogue, we have determined a revised estimate of the local white dwarf space density. We repeated the procedure 
outlined in Hollands et al. (2018b), where the main difference is the four previously missing objects from DR2 now present in EDR3 as described above. This acts to increase the volume-averaged detection efficiency to $98.3_{-1.2}^{+0.6}$ per cent (compared with $96.0_{-1.6}^{+1.3}$ per cent for DR2), corresponding to an effective volume sampled by Gaia of $32950_{-390}^{+230} \mathrm{pc}^{3}$ (from $32170_{-540}^{+420} \mathrm{pc}^{3}$ for DR2). This increase in effective volume is compensated by for increased number of objects detected in EDR3, thus leading to a revised space density of $4.47 \pm 0.37 \times 10^{-3} \mathrm{pc}^{-3}-$ almost unchanged from our DR2 estimate of $4.49 \pm 0.38 \times 10^{-3} \mathrm{pc}^{-3}$. The approach of Hollands et al. (2018b) also yields the distribution for the number of white dwarfs that remain undiscovered within 20 pc. As with DR2, the most likely value is zero, but with its probability increased from 28 per cent to 37 per cent. The median of this distribution has decreased from 2 to 1 , i.e. there is only a 40 per cent probability that more than one white dwarf remains undetected within the $20 \mathrm{pc}$ volume.

\subsubsection{0 pc volume sample}

There are 33 new sources in our catalogue and within $40 \mathrm{pc}$ that were not in Gentile Fusillo et al. (2019) and within the same volume. Nine are known white dwarfs that had incomplete or missing data in DR2 such that they could not be reliably identified as white dwarfs, while five are known white dwarfs that have moved from $>40$ to $<40 \mathrm{pc}$. All 14 known white dwarfs have $P_{\mathrm{WD}}>0.75$. The faintest confirmed white dwarf in the $40 \mathrm{pc}$ sample is $G=19.61$, and we note that only two new candidates are fainter.

The majority of the 19 remaining new sources are either wide companions to bright known main-sequence stars (with separation from 5 to 20 arcsec), or are near bright background sources, or are in crowded fields. We suspect that Gaia colours may be unreliable for sources close to bright companions of magnitude $G \approx 6-12$. Consequently we warn users that some of these new white dwarf candidates may potentially be misidentified low-mass stars with poor background subtraction or contamination from a companion, although a few could also be genuine new Sirius-like systems.

As a consequence, it is difficult to estimate the number of genuine new EDR3 white dwarfs within $40 \mathrm{pc}$, but it could be only a handful. To give the catalogue users some indication of which objects may be affected by the presence of a bright neighbour, we provide a BRIGHT_NEIGHBOUR flag. This flag is given to all stars with a neighbour $\simeq 5$ mag brighter than themselves at a separation of 5 arcsec or less. A number of extremely bright stars are not included at all in Gaia EDR3, so to compute our BRIGHT_NEIGHBOUR flag we also performed a cross-match with the Tycho- 2 catalogue of bright stars (Høg et al. 2000).

No confirmed $40 \mathrm{pc}$ white dwarf identified in DR2 is missing from our new catalogue, but three confirmed white dwarfs have moved from within $40 \mathrm{pc}$ to outside of the volume: WD J102459.83+044610.50, WD J065722.88+024100.84, and WD J214810.74-562613.14. There are 18 additional highprobability candidates from DR2 that are gone. None are known to be white dwarfs but 10 were confirmed as main-sequence stars in Tremblay et al. (2020). Finally, 162 low-probability white dwarf candidates from Gentile Fusillo et al. (2019) are now entirely gone from the $40 \mathrm{pc}$ sample. None of them had been identified as genuine white dwarf by spectroscopic follow-up.

Now we focus our attention on the northern 40 pc Gaia sample with already high spectroscopic completeness, with all membership changes listed in Table A1. The full EDR3 Northern hemisphere 40 pc sample can also be queried via the VizieR catalogue access tool as a direct update of the tables presented in McCleery et al. (2020).

Of the 521 spectroscopically confirmed Gaia DR2 white dwarfs from McCleery et al. (2020), two have now moved beyond $40 \mathrm{pc}$ as discussed above (category B of Table A1). This is compensated by 12 previously known white dwarfs moving in or now having full Gaia solutions (category A of Table A1), for a new total of 531 spectroscopically confirmed white dwarfs. However, several externally confirmed $40 \mathrm{pc}$ members are still missing from our EDR3 catalogue (categories C and D of Table A1).

Three unobserved high-probability DR2 candidates (category E of Table A1) are still strong candidates in EDR3 and likely white dwarfs. Furthermore, five unobserved low-probability white dwarf candidates in table A2 of McCleery et al. (2020) are now highprobability candidates in our catalogue (category E of Table A1), hence likely white dwarfs. Even accounting for the few new EDR3 candidates that may be genuine white dwarfs, we estimate that only $\simeq 10$ white dwarfs within 40 pc north have not yet received spectroscopic follow-up, corresponding to a spectroscopic completeness of $>98$ per cent.

\subsection{Change in white dwarf stellar parameters}

To characterize changes in derived white dwarf fundamental parameters between EDR3 and DR2, we rely on the well-characterized northern 40 pc sample. We compare the published DR2 parameters from table A1 of McCleery et al. (2020) with the updated EDR3 VizieR version of the same table (see Section 7.5.2). Fig. 13 (top panel) shows the resulting EDR3 $T_{\text {eff }}$ versus mass distribution, which is very similar to that found in McCleery et al. (2020) using DR2 and the same model atmospheres. To illustrate this we plot EDR3 versus DR2 differences in $T_{\text {eff }}$ and mass in the middle panels of Fig. 13. It demonstrates that despite changes in passbands and colour calibration (Riello et al. 2021), there are almost negligible systematic changes between DR2 and EDR3 parameters. While $T_{\text {eff }}$ depends almost only on Gaia colours, mass is sensitive to both changes in colours $\left(T_{\text {eff }}\right)$ and parallax. The $\Delta M$ scatter is almost twice as large as the $\Delta T_{\text {eff }}$ scatter, therefore suggesting that changes in colours and parallax contribute roughly equally to changes in mass (or $\log g$ ). Finally, the bottom panel of Fig. 13 compares absolute changes in mass between catalogues and our formal mass error bars that are based solely on catalogued Gaia data errors. The median change in mass is $0.57 \sigma$, which demonstrates that despite Gaia error bars being very small, they appear to be a good representation of the precision of the data. Nevertheless, this does not account for possible systematic issues in both DR2 and EDR3 that could impact the accuracy of Gaia atmospheric parameters (see Section 7.7).

We note that reddening is very small and essentially negligible for the $40 \mathrm{pc}$ sample. This is not the case for our overall EDR3 catalogue, where stellar parameters are also modified by our newly adopted extinction maps.

\subsection{Calibration of Gaia EDR3 colours}

Cukanovaite et al. (2021) tested the calibration of Gaia DR2 colours, by comparing spectroscopically and photometrically derived parameters for various samples of DA and DB white dwarfs. In this section, we reproduce the test performed in Cukanovaite et al. (2021), using our stellar parameters based on Gaia EDR3 photometry (see Section 4) and spectroscopically derived parameters recovered from various other studies. The DA white dwarfs used in this comparison are from two samples: the SDSS sample from Tremblay et al. (2019a) 

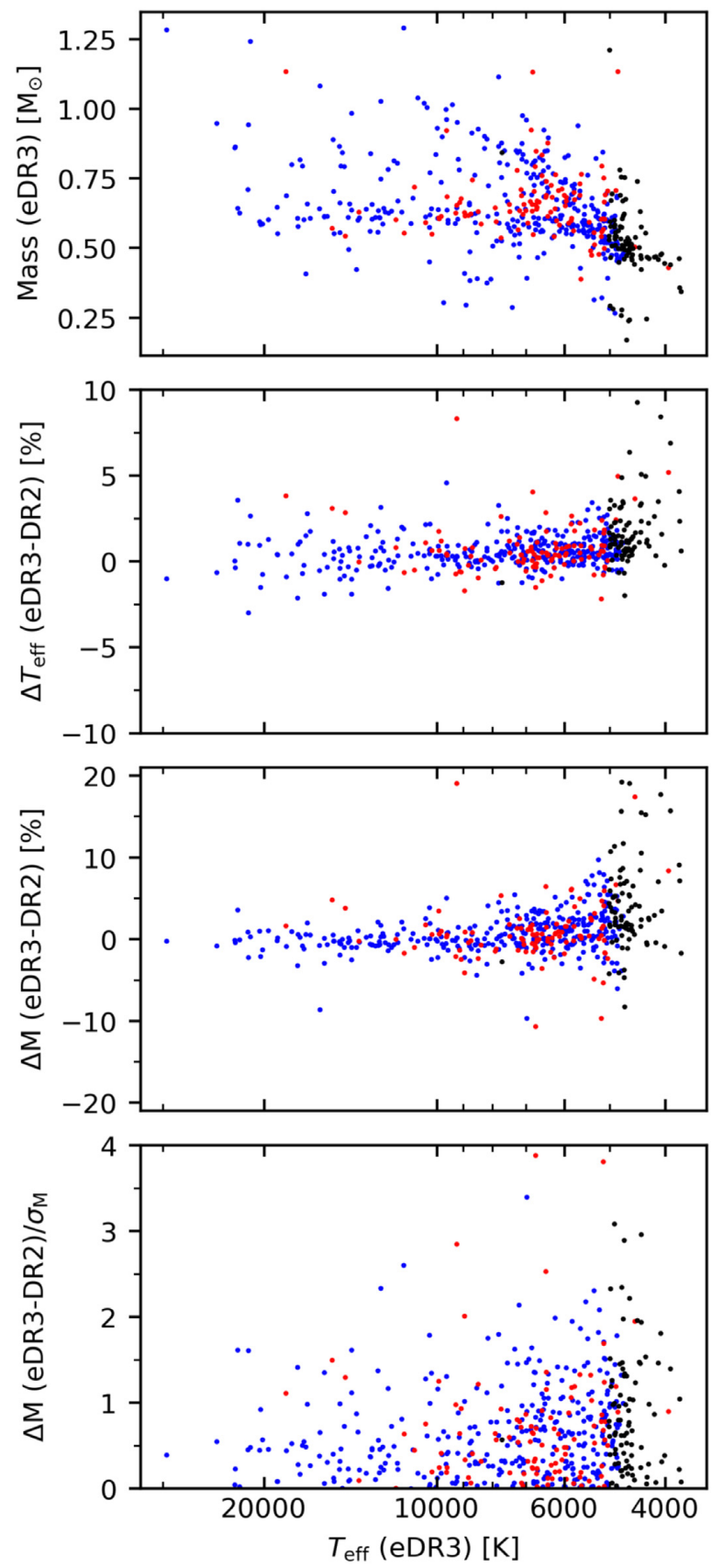

Figure 13. Top panel: EDR $3 T_{\text {eff }}$ versus mass distribution for the northern $40 \mathrm{pc}$ white dwarf sample. The sample is similar to that of McCleery et al. (2020) but with updated EDR3 parameters and minor changes in membership (see Table A1). Spectrally identified H-rich atmospheres are shown in blue, He-rich atmospheres in red, and unconstrained compositions in black (cool DC white dwarfs and unobserved candidates). Top-middle panel: difference in $T_{\text {eff }}$ (per cent) between EDR3 and DR2. Bottom-middle panel: difference in mass (per cent) between EDR3 and DR2. Bottom panel: absolute difference in mass divided by EDR3 mass uncertainty.

and the Gianninas, Bergeron \& Ruiz (2011) sample. The latter sample has been corrected for 3D effects by Tremblay et al. (2019a). The DB samples are from Genest-Beaulieu \& Bergeron (2019b) and Rolland, Bergeron \& Fontaine (2018), with additional correction for 3D effects from Cukanovaite et al. (2021). The Genest-Beaulieu \& Bergeron (2019b) sample has also been corrected to put van der Waals broadening on the same scale as the other samples. For a more detailed discussion on these corrections, see Cukanovaite et al. (2021).

We cross-matched the coordinates of all white dwarfs in the spectroscopic samples with our Gaia EDR3 catalogue and recovered the photometrically derived effective temperatures and surface gravities. Only a small percentage of the white dwarfs did not have a match in our catalogue because of missing or unreliable Gaia EDR3 parallaxes or colours.

For all successful matches, the differences in spectroscopic and photometric effective temperatures and surface gravities were calculated. For the final comparison, we removed all white dwarfs that had absolute differences larger than 30 per cent. This was done to remove any physical outliers, such as unresolved binaries. This clipping affects only a very small percentage of objects, with the exception of the Rolland et al. (2018) sample, where at very low effective temperatures the high-log $g$ problem is apparent when the removal is not performed. Nevertheless, this does not change our conclusions. For a full overview of the Rolland et al. (2018) sample without the removal of outliers, see fig. 15 in Cukanovaite et al. (2021).

Fig. 14 displays the results of our comparison of spectroscopically and photometrically derived parameters. The plot shows the median difference in bins of $2000 \mathrm{~K}$ for $T_{\text {eff }}<20000 \mathrm{~K}$, and in bins of $5000 \mathrm{~K}$ for $T_{\text {eff }}>20000 \mathrm{~K}$. By comparing this figure with figs 14 and 15 in Cukanovaite et al. (2021), it is clear that the offsets seen in the spectroscopically and photometrically derived parameters are very similar for Gaia DR2 and EDR3.

$\mathrm{A} \simeq 5$ per cent offset in effective temperature can be seen around $20000 \mathrm{~K}$. Analogously to what was concluded by Cukanovaite et al. (2021), we attribute this to issues with Gaia colour calibrations.

In particular, we note that the spectrophotometric calibration of the Space Telescope Imaging Spectrograph (STIS) onboard the Hubble Space Telescope (HST) is tied to the spectroscopic parameters of three white dwarfs that are also part of the Gianninas et al. (2011) sample adopted here. Therefore, the systematic $T_{\text {eff }}$ offset observed in Fig. 14 suggests that Gaia EDR3 is still not entirely consistent with HST/STIS calibration (Maíz Apellániz \& Weiler 2018).

Other explanations could include issues with the calibration of the surveys from which the spectroscopic samples were derived, such as SDSS. However, SDSS and non-SDSS spectroscopic samples show similar offsets. Another explanation could be issues with the microphysics of model spectra, such as prescriptions of line broadening. We believe this to be a less likely explanation because line broadening theories for DA and DB white dwarfs are entirely different and have different temperature dependencies. Thus, it is difficult to see how the offset could be so similar between the two types of white dwarf samples.

\section{CONCLUSION}

We present a catalogue of white dwarf candidates selected from Gaia EDR3. Starting from the entire 1.47 billion sources with an astrometric solution in EDR3, we defined a number of cuts in colour, absolute magnitude, and various Gaia quality parameters to broadly isolate the white dwarf locus in the $\mathrm{H}-\mathrm{R}$ diagram and remove objects with unreliable Gaia measurements. This selection resulted in a sample of 1280266 EDR3 sources. Following the same methodology described for DR2 in Gentile Fusillo et al. (2019) we then made use of a sample SDSS spectroscopically classified objects to map the distribution of white dwarfs and contaminant objects in $G_{\mathrm{BP}}-G_{\mathrm{RP}}$ colour $-G_{\mathrm{abs}}$ space and calculate probabilities of being $a$ white dwarf $\left(P_{\mathrm{WD}}\right)$ for all objects in our sample. Coupled with 

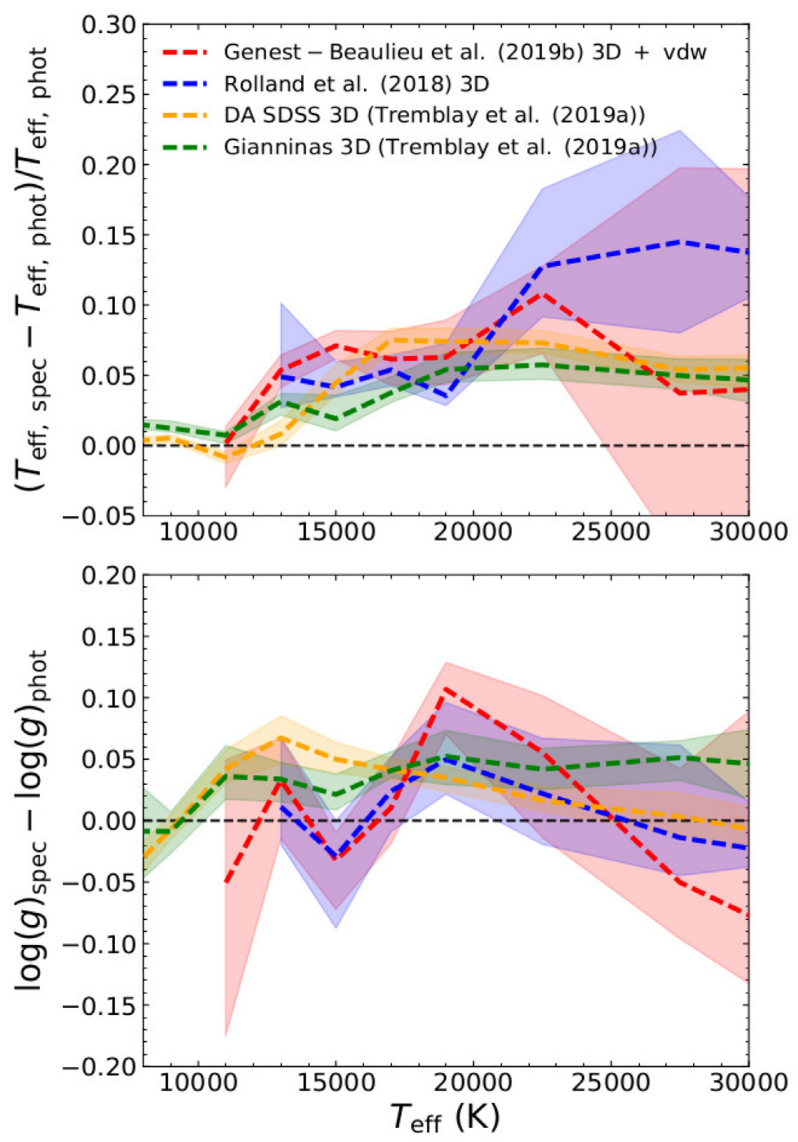

Figure 14. A comparison between EDR $3 T_{\text {eff }}$ and $\log g$ values calculated in this paper and corresponding spectroscopically derived parameters from literature. The spectroscopic parameters for the two DA samples are from Tremblay et al. (2019a). The DB spectroscopic parameters are from GenestBeaulieu \& Bergeron (2019b) and Rolland et al. (2018), and have been corrected for 3D effects by Cukanovaite et al. (2021). In the case of GenestBeaulieu \& Bergeron (2019b), the sample has also been corrected for van der Waals effect (see Cukanovaite et al. 2021). The dashed lines indicate the medians for the various samples. The coloured areas represent the error on the median found by bootstrapping. The median difference was calculated in bins of $2000 \mathrm{~K}$ for $T_{\text {eff }}<20000 \mathrm{~K}$ and in bins of $5000 \mathrm{~K}$ for $T_{\text {eff }}>20000 \mathrm{~K}$.

Gaia quality flags these $P_{\mathrm{WD}}$ values allow to flexibly select samples of white dwarf candidates compromising between completeness and contamination according to the users' specific goals. For general purpose we recommend a cut at $P_{\mathrm{WD}}>0.75$, which selects a sample of 359073 objects. This subset includes the vast majority of the white dwarfs in the catalogue, with minimal level of contamination. We also utilized state-of-the-art 3D extinction maps to correct the three Gaia photometric bands for reddening, and provide stellar parameters ( $T_{\text {eff }}$, $\log g$, and mass) obtained by fitting Gaia photometry and parallax for all objects with $P_{\mathrm{WD}}>0.70$. In addition to stellar parameters and $P_{\mathrm{WD}}$ values, our catalogue includes a number of other columns not available in the main Gaia EDR3 archival distribution, which can be used to further characterize any sample of white dwarf candidates selected. For example, the parameter EXCESS_FLUX_ERROR is an indicator of the variability in the flux of an object during the multiple Gaia observations.

To assess the overall completeness of the main Gaia EDR3 catalogue of white dwarfs, we have used an independent sample of 60739 white dwarf candidates selected from SDSS on the basis of their colours and proper motions. We estimate our Gaia EDR3 catalogue to be between 67 and 93 per cent complete for white dwarfs with $G \leq 20$ and $T_{\text {eff }}>7000 \mathrm{~K}$, at high Galactic latitudes $(|b|>$ $20^{\circ}$ ). Together with the main catalogue of white dwarf candidates we also provide an extension containing objects with unreliable parallax measurements (PARALLAX_OVER_ERROR $<1$ ), but which can be identified as white dwarfs using their RPM. An additional $\simeq 10200$ white dwarfs can be recovered from this RPM extension.

We also cross-matched both the main catalogue and the RPM extension with the entire spectral library of SDSS DR16, retrieving a total of 39223 spectra corresponding to 29727 objects. All spectra were visually inspected and classified according to their spectral type. We provide this Gaia-SDSS spectroscopic catalogue in a separate distribution from the main catalogue of white dwarf candidates.

The catalogue presented in this paper is by far the largest collection of white dwarfs published to date exceeding the size of the largest DR2-based catalogue by nearly 100000 objects. With EDR3 virtually every part of the sky now has Gaia parameters robust enough to identify all white dwarfs with $G \leq 20$. We therefore do not expect significant further improvements in the overall number of known white dwarfs until reliable astrometry for fainter stars will become available thanks to next generation observatories (e.g. Vera Rubin Observatory; Ivezić et al. 2019). However, to date only a small fraction of these Gaia white dwarfs have received any spectroscopic observations. This type of follow-up is fundamental in order to study white dwarfs in detail, both as individual objects and as a stellar population. With new large multifibre spectroscopic facilities now beginning operation in both hemispheres (e.g. WEAVE, 4MOST, DESI, SDSS-V; Dalton et al. 2014; de Jong et al. 2014; DESI Collaboration 2016; Kollmeier et al. 2017), we are on the verge of a revolution in observational astronomy, and spectroscopic coverage of every known white dwarf may become a reality in the not-too-distant future. Our $P_{\mathrm{WD}}$ values combined with stellar parameters provided, and the various Gaia metrics, allow to construct well-defined white dwarf samples for any spectroscopic follow-up campaign, making the EDR3 catalogue of white dwarf candidates presented here a key resource for any future white dwarf study.

\section{ACKNOWLEDGEMENTS}

P-ET has received funding from the European Research Council under the European Union's Horizon 2020 Framework Programme no. 677706 (WD3D). RL acknowledges access to results from the EXPLORE project prior to publication. EXPLORE has received funding from the European Union's Horizon 2020 Framework Programme under grant agreement no. 101004214. This work has made use of data from the European Space Agency (ESA) mission Gaia (https://www.cosmos.esa.int/gaia), processed by the Gaia Data Processing and Analysis Consortium (DPAC, https://www.cosm os.esa.int/web/gaia/dpac/consortium). Funding for the DPAC has been provided by national institutions, in particular the institutions participating in the Gaia Multilateral Agreement. Funding for the Sloan Digital Sky Survey IV has been provided by the Alfred P. Sloan Foundation, the U.S. DOE Office of Science, and the Participating Institutions.

SDSS-IV acknowledges support and resources from the Center for High Performance Computing at the University of Utah. The SDSS website is www.sdss.org.

SDSS-IV is managed by the Astrophysical Research Consortium for the Participating Institutions of the SDSS Collaboration including the Brazilian Participation Group, the Carnegie Institution for Science, Carnegie Mellon University, Center for Astrophysics | Harvard \& Smithsonian, the Chilean Participation Group, the French 
Participation Group, Instituto de Astrofísica de Canarias, The Johns Hopkins University, Kavli Institute for the Physics and Mathematics of the Universe (IPMU)/University of Tokyo, the Korean Participation Group, Lawrence Berkeley National Laboratory, Leibniz Institut für Astrophysik Potsdam (AIP), Max-Planck-Institut für Astronomie (MPIA Heidelberg), Max-Planck-Institut für Astrophysik (MPA Garching), Max-Planck-Institut für Extraterrestrische Physik (MPE), National Astronomical Observatories of China, New Mexico State University, New York University, University of Notre Dame, Observatário Nacional/MCTI, The Ohio State University, Pennsylvania State University, Shanghai Astronomical Observatory, United Kingdom Participation Group, Universidad Nacional Autónoma de México, University of Arizona, University of Colorado Boulder, University of Oxford, University of Portsmouth, University of Utah, University of Virginia, University of Washington, University of Wisconsin, Vanderbilt University, and Yale University.

This work was funded by the Deutsche Forschungsgemeinschaft (DFG, German Research Foundation) - Project ID 138713538 - SFB 881 ('The Milky Way System').

\section{DATA AVAILABILITY}

The catalogues presented in this work can be downloaded from this link. They will also be made available via the VizieR Service for Astronomical Catalogues. All additional data underlying this paper are publicly available from the relevant survey archives or will be shared on reasonable request to the corresponding author.

\section{REFERENCES}

Abril J., Schmidtobreick L., Ederoclite A., López-Sanjuan C., 2020, MNRAS, 492, L40

Ahumada R. et al., 2019, ApJS, 249, 3

Bailer-Jones C. A. L., Rybizki J., Fouesneau M., Demleitner M., Andrae R., 2021, AJ, 161, 147

Bauer E. B., Schwab J., Bildsten L., Cheng S., 2020, ApJ, 902, 93

Bédard A., Bergeron P., Brassard P., Fontaine G., 2020, ApJ, 901, 93

Bergeron P., 2001, ApJ, 558, 369

Bergeron P. et al., 2011, ApJ, 737, 28

Bergeron P., Dufour P., Fontaine G., Coutu S., Blouin S., Genest-Beaulieu C., Bédard A., Rolland B., 2019, ApJ, 876, 67

Blouin S., Dufour P., 2019, MNRAS, 490, 4166

Blouin S., Daligault J., Saumon D., 2021, ApJ, 911, L5

Bohlin R. C., Gordon K. D., Tremblay P. E., 2014, PASP, 126, 711

Camisassa M. E., Althaus L. G., Torres S., Córsico A. H., Rebassa-Mansergas A., Tremblay P.-E., Cheng S., Raddi R., 2021, A\&A, 649, L7

Capitanio L., Lallement R., Vergely J. L., Elyajouri M., Monreal-Ibero A., 2017, A\&A, 606, A65

Chandra V., Hwang H.-C., Zakamska N. L., Cheng S., 2020, ApJ, 899, 146

Cheng S., Cummings J. D., Ménard B., 2019, ApJ, 886, 100

Coutu S., Dufour P., Bergeron P., Blouin S., Loranger E., Allard N. F., Dunlap B. H., 2019, ApJ, 885, 74

Cukanovaite E., Tremblay P.-E., Bergeron P., Freytag B., Ludwig H.-G., Steffen M., 2021, MNRAS, 501, 5274

Dalton G. et al., 2014, in Ramsay S. K., McLean I. S., Takami H., eds, Proc. SPIE Vol. 9147, Ground-Based and Airborne Instrumentation for Astronomy V. SPIE, Bellingham, p. 91470L

de Jong R. S. et al., 2014, in Ramsay S. K., McLean I. S., Takami H., eds, Proc. SPIE Vol. 9147, Ground-Based and Airborne Instrumentation for Astronomy V. SPIE, Bellingham, p. 91470M

Dennihy E. et al., 2020, ApJ, 905, 5

DESI Collaboration, 2016, preprint (arXiv:1611.00036)

Dufour P., Blouin S., Coutu S., Fortin-Archambault M., Thibeault C., Bergeron P., Fontaine G., 2017, in Tremblay P.-E., Gaensicke B., Marsh
T., eds, ASP Conf. Ser. Vol. 509, 20th European White Dwarf Workshop. Astron. Soc. Pac., San Francisco, p. 3

Eisenstein D. J. et al., 2006, ApJS, 167, 40

El-Badry K., Rix H.-W., Weisz D. R., 2018, ApJ, 860, L17

Fabricius C. et al., 2021, A\&A, 649, A5

Fontaine G., Brassard P., Bergeron P., 2001, PASP, 113, 409

Gaia Collaboration, 2021a, A\&A, 649, A1

Gaia Collaboration, 2021b, A\&A, 649, A6

Gaia Collaboration, 2021c, A\&A, 649, A7

Gänsicke B. T., Schreiber M. R., Toloza O., Fusillo N. P. G., Koester D., Manser C. J., 2019, Nature, 576, 61

Genest-Beaulieu C., Bergeron P., 2019a, ApJ, 871, 169

Genest-Beaulieu C., Bergeron P., 2019b, ApJ, 882, 106

Gentile Fusillo N. P., Gänsicke B. T., Greiss S., 2015, MNRAS, 448, 2260

Gentile Fusillo N. P. et al., 2019, MNRAS, 482, 4570

Gentile Fusillo N. P., Tremblay P.-E., Bohlin R. C., Deustua S. E., Kalirai J. S., 2020, MNRAS, 491, 3613

Gentile Fusillo N. P. et al., 2021, MNRAS, 504, 2707

Giammichele N., Bergeron P., Dufour P., 2012, ApJS, 199, 29

Gianninas A., Bergeron P., Ruiz M. T., 2011, ApJ, 743, 138

Gianninas A., Kilic M., Brown W. R., Canton P., Kenyon S. J., 2015, ApJ, 812,167

Guidry J. A. et al., 2021, ApJ, 912, 125

Hansen B. M. S., 1998, Nature, 394, 860

Harris H. C. et al., 2006, AJ, 131, 571

Hirsch L. A. et al., 2019, ApJ, 878, 50

Høg E. et al., 2000, A\&A, 355, L27

Holberg J. B., Oswalt T. D., Sion E. M., 2002, ApJ, 571, 512

Hollands M. A., Gänsicke B. T., Koester D., 2018a, MNRAS, 477, 93

Hollands M. A., Tremblay P. E., Gänsicke B. T., Gentile-Fusillo N. P., Toonen S., 2018b, MNRAS, 480, 3942

Hollands M. A. et al., 2020, Nat. Astron., 4, 663

Hollands M. A., Tremblay P.-E., Gänsicke B. T., Koester D., Gentile-Fusillo N. P., 2021, Nat. Astron., 5, 451

Inight K., Gänsicke B. T., Breedt E., Marsh T. R., Pala A. F., Raddi R., 2021, MNRAS, 504, 2420

Ivanova A., Lallement R., Vergely J. L., Hottier C., 2021, A\&A, 652, A22

Ivezić Ž. et al., 2019, ApJ, 873, 111

Jiménez-Esteban F. M., Torres S., Rebassa-Mansergas A., Skorobogatov G., Solano E., Cantero C., Rodrigo C., 2018, MNRAS, 480, 4505

Jones E. M., 1972, ApJ, 177, 245

Kaiser B. C., Clemens J. C., Blouin S., Dufour P., Hegedus R. J., Reding J. S., Bédard A., 2021, Science, 371, 168

Kawka A., Vennes S., 2006, ApJ, 643, 402

Kawka A., Vennes S., Ferrario L., 2020, MNRAS, 491, L40

Kilic M. et al., 2006, AJ, 131, 582

Kilic M., Bergeron P., Blouin S., Bédard A., 2021, MNRAS, 503, 5397

Kilic M., Bergeron P., Kosakowski A., Brown W. R., Agüeros M. A., Blouin S., 2020, ApJ, 898, 84

Koester D., 2013, in Oswalt T. D., Barstow M. A., eds, Planets, Stars and Stellar Systems. Springer, Dordrecht, p. 559

Kollmeier J. A. et al., 2017, preprint (arXiv:1711.03234)

Kowalski P. M., Saumon D., 2006, ApJ, 651, L137

Lallement R., Vergely J. L., Valette B., Puspitarini L., Eyer L., Casagrande L., 2014, A\&A, 561, A91

Lallement R., Babusiaux C., Vergely J. L., Katz D., Arenou F., Valette B., Hottier C., Capitanio L., 2019, A\&A, 625, A135

Lam M. C. et al., 2019, MNRAS, 482, 715

Leggett S. K. et al., 2018, ApJS, 239, 26

Limoges M. M., Bergeron P., Lépine S., 2015, ApJS, 219, 19

Lindegren L. et al., 2018, A\&A, 616, A2

Lindegren L. et al., 2021, A\&A, 649, A2

López-Sanjuan C. et al., 2019, A\&A, 631, A119

Maíz Apellániz J., Weiler M., 2018, A\&A, 619, A180

McCleery J. et al., 2020, MNRAS, 499, 1890

Melis C., Klein B., Doyle A. E., Weinberger A. J., Zuckerman B., Dufour P., 2020, ApJ, 905, 56 
Mukadam A. S. et al., 2013, ApJ, 771, 17

Narayan G. et al., 2019, ApJS, 241, 20

Ourique G., Romero A. D., Kepler S. O., Koester D., Amaral L. A., 2019, MNRAS, 482, 649

Pala A. F. et al., 2020, MNRAS, 494, 3799

Queiroz A. B. A. et al., 2020, A\&A, 638, A76

Remy Q., Grenier I. A., Marshall D. J., Casandjian J. M., 2018, A\&A, 616, A71

Riello M. et al., 2021, A\&A, 649, A3

Rolland B., Bergeron P., Fontaine G., 2018, ApJ, 857, 56

Rybizki J. et al., 2021, preprint (arXiv:2101.11641)

Sanders J. L., Das P., 2018, MNRAS, 481, 4093

Schlafly E. F., Finkbeiner D. P., 2011, ApJ, 737, 103

Schlegel D. J., Finkbeiner D. P., Davis M., 1998, ApJ, 500, 525

Serenelli A. M., Althaus L. G., Rohrmann R. D., Benvenuto O. G., 2001, MNRAS, 325, 607

Sion E. M., Greenstein J. L., Landstreet J. D., Liebert J., Shipman H. L., Wegner G. A., 1983, ApJ, 269, 253
Sion E. M., Holberg J. B., Oswalt T. D., McCook G. P., Wasatonic R., Myszka J., 2014, AJ, 147, 129

Tian H.-J. et al., 2017, ApJS, 232, 4

Torres S., Cantero C., Rebassa-Mansergas A., Skorobogatov G., JiménezEsteban F. M., Solano E., 2019, MNRAS, 485, 5573

Torres S., Rebassa-Mansergas A., Camisassa M. E., Raddi R., 2021, MNRAS, 502, 1753

Tremblay P.-E., Bergeron P., Gianninas A., 2011, ApJ, 730, 128

Tremblay P.-E., Kalirai J. S., Soderblom D. R., Cignoni M., Cummings J., 2014, ApJ, 791, 92

Tremblay P.-E., Cukanovaite E., Gentile Fusillo N. P., Cunningham T., Hollands M. A., 2019a, MNRAS, 482, 5222

Tremblay P.-E. et al., 2019b, Nature, 565, 202

Tremblay P. E. et al., 2020, MNRAS, 497, 130

van Horn H. M., 1968, ApJ, 151, 227

van Leeuwen F., 2007, A\&A, 474, 653

Vergely J.-L., Valette B., Lallement R., Raimond S., 2010, A\&A, 518, A31

Zuckerman B., Becklin E. E., Macintosh B. A., Bida T., 1997, AJ, 113, 764

\section{APPENDIX A: CHANGES IN THE NORTHERN HEMISPHERE 40 PC SAMPLE}

Table A1. Changes in the northern $40 \mathrm{pc}$ white dwarf sample between DR2 and EDR3. This excludes 519 spectroscopically confirmed white dwarfs that are part of the sample in both DR2 and EDR3 (see table A1 of McCleery et al. 2020).

\begin{tabular}{|c|c|c|c|c|c|c|c|}
\hline Gaia DR3 ID & $\begin{array}{c}\text { WD name } \\
\text { (confirmed WDs only) }\end{array}$ & $P_{\mathrm{WD}}$ & $\begin{array}{l}\text { RA } \\
\left({ }^{\circ}\right)\end{array}$ & $\begin{array}{c}\text { Dec. } \\
\left({ }^{\circ}\right)\end{array}$ & $\begin{array}{c}\varpi\left(\sigma_{\varpi}\right) \\
(\mathrm{mas})\end{array}$ & $\begin{array}{c}G\left(\sigma_{\mathrm{G}}\right) \\
(\mathrm{mag})\end{array}$ & Comment \\
\hline \multicolumn{8}{|c|}{ (A) New EDR3 catalogue entries } \\
\hline 2545505281002947200 & WD $0011+000$ & 0.995 & 3.415 & 0.322 & $25.002(0.041)$ & $15.336(0.002)$ & SpT: DA (Limoges et al. 2015), moved to $40 \mathrm{pc}$ \\
\hline 307323228064848512 & WD $0108+277$ & 0.748 & 17.685 & 27.970 & $26.312(0.082)$ & $16.153(0.003)$ & SpT: DAZ (Kawka \& Vennes 2006), incomplete entry in DR2 \\
\hline 3320184202856435840 & WD $0553+053$ & 0.995 & 89.104 & 5.359 & $123.198(0.017)$ & $13.969(0.002)$ & SpT: DAH (Limoges et al. 2015), incomplete entry in DR2 \\
\hline 1146403741412820864 & WD J102203.66+824310.00 & 0.991 & 155.505 & 82.718 & $25.113(0.092)$ & $17.899(0.003)$ & SpT: DA (Tremblay et al. 2020), moved to $40 \mathrm{pc}$ \\
\hline 3883918657822146944 & - & 0.994 & 157.361 & 12.959 & $27.751(0.166)$ & $17.460(0.003)$ & New EDR3 white dwarf candidate \\
\hline 3978879594463300992 & WD $1121+216$ & 0.992 & 171.049 & 21.359 & $68.041(0.028)$ & $14.124(0.002)$ & SpT: DA (Limoges et al. 2015), incomplete entry in DR2 \\
\hline 3920187251456610816 & WD $1153+135$ & 0.977 & 179.048 & 13.265 & $28.115(0.090)$ & $17.401(0.002)$ & SpT: DC (Leggett et al. 2018), incomplete entry in DR2 \\
\hline 3905335598144227200 & WD $1218+095$ & 1.000 & 185.201 & 9.235 & $26.681(0.346)$ & $19.606(0.005)$ & SpT: DC (Leggett et al. 2018) incomplete entry in DR2 \\
\hline 3713594960831605760 & WD $1334+039$ & 0.996 & 204.116 & 3.674 & $119.756(0.030)$ & $14.376(0.002)$ & SpT: DA (Limoges et al. 2015), missing from DR2 \\
\hline 1336988963803208192 & - & 0.941 & 259.981 & 36.657 & $28.539(0.051)$ & $16.953(0.003)$ & Suspicious candidate, $4 \operatorname{arcsec}$ wide companion to $\mathrm{M}$ dwarf \\
\hline 4512265810525783680 & - & 0.993 & 281.752 & 18.185 & $34.486(0.037)$ & $15.332(0.002)$ & Suspicious candidate, 20 arcsec wide companion to HD 173880 \\
\hline 4539227892919675648 & WD J184733.18+282057.54 & 0.999 & 281.889 & 28.347 & $25.001(0.120)$ & $18.439(0.003)$ & SpT: DC (Tremblay et al. 2020), moved to $40 \mathrm{pc}$ \\
\hline 4288942973032203904 & - & 1.000 & 290.526 & 2.553 & $25.377(0.265)$ & $19.119(0.004)$ & SpT: DZ (Tremblay et al. 2020), moved to $40 \mathrm{pc}$ \\
\hline 2701893698904233216 & LP $578-24$ & 0.986 & 325.726 & 8.090 & $33.449(0.086)$ & $17.052(0.002)$ & SpT: DA (Limoges et al. 2015), incomplete entry in DR2 \\
\hline 2730707260103011712 & WD $2220+121.1$ & 0.999 & 335.644 & 12.362 & $25.996(0.218)$ & $18.366(0.003)$ & SpT: DC (Kilic et al. 2006), incomplete entry in DR2 \\
\hline \multicolumn{8}{|c|}{ (B) DR2 white dwarf members moved out of the sample } \\
\hline 3127761765259717632 & WD J065722.88+024100.84 & 0.997 & 104.345 & 2.682 & $24.879(0.056)$ & $16.096(0.02)$ & SpT: DC (Limoges et al. 2015) \\
\hline 3860381618565361024 & WD J102459.83+044610.50 & 0.997 & 156.248 & 4.769 & $23.204(0.046)$ & $14.214(0.02)$ & SpT: DA (Gianninas et al. 2011) \\
\hline \multicolumn{8}{|c|}{ (C) Confirmed northern $40 \mathrm{pc}$ white dwarfs missing from DR2 and EDR3 } \\
\hline 975968340912517248 & WD $0727+482 \mathrm{~A}$ & - & 112.695 & 48.168 & - & $15.063(0.02)$ & $\begin{array}{l}\text { SpT: DA (Limoges et al. 2015), } \varpi=88.723 \pm 0.029 \text { (Gaia EDR3 } \\
\text { companion G107-69) }\end{array}$ \\
\hline 975968340910692608 & WD $0727+482 B$ & - & 112.695 & 48.168 & - & $15.251(0.02)$ & $\begin{array}{l}\text { SpT: DA (Limoges et al. 2015), } \varpi=88.723 \pm 0.029 \text { (Gaia EDR3 } \\
\text { companion G107-69) }\end{array}$ \\
\hline- & WD $0736+053$ & - & - & - & - & - & SpT: DQZ (Limoges et al. 2015), $\varpi=284.56 \pm 1.26$ (van Leeuwen 2007) \\
\hline 3817534337626005632 & WD $1120+073$ & - & 170.881 & 7.022 & - & $17.511(0.02)$ & $\begin{array}{l}\text { SpT: DC (Limoges et al. 2015), } \varpi=27.025 \pm 0.469 \text { (Gaia EDR3 } \\
\text { companion LP 552-48) }\end{array}$ \\
\hline 3701290326205270528 & WD $1214+032$ & - & 184.213 & 2.968 & $42.772(0.042)$ & $15.330(0.02)$ & SpT: DA (Limoges et al. 2015), no colour information in EDR3 \\
\hline 1362295082910739840 & HD 159062B & - & 262.568 & 47.402 & - & $16.745(0.02)$ & $\begin{array}{l}\text { SpT: G9V + WD (Hirsch et al. 2019), } \varpi=46.185 \pm 0.014 \text { (Gaia } \text { EDR3 } \\
\text { companion HD 159062) }\end{array}$ \\
\hline 2274076301516712704 & WD 2126+734B & - & 321.741 & 73.643 & $44.909(0.069)$ & $16.511(0.02)$ & $\begin{array}{l}\text { SpT: DC (Zuckerman et al. 1997), missing from catalogue due to large } \\
\text { BP/RP excess factor }\end{array}$ \\
\hline 1962707287281651712 & PM J22105+4532 & - & 332.643 & 45.542 & $27.858(0.078)$ & $17.146(0.02)$ & SpT: DC (Limoges et al. 2015), no colour information in EDR3 \\
\hline \multicolumn{8}{|c|}{ (D) Unresolved binaries missing from our catalogues } \\
\hline 1005873614080407296 & LHS 1817 & - & 91.375 & 60.819 & $61.426(0.053)$ & $12.296(0.03)$ & SpT: M4.5V + WD (McCleery et al. 2020) \\
\hline 3845263368043086080 & WD $0911+023$ & - & 138.591 & 2.312 & $27.070(0.616)$ & $3.88(0.05)$ & SpT: B9.5V + WD (McCleery et al. 2020) \\
\hline 1548104507825815296 & WD $1213+528$ & - & 183.933 & 52.516 & $34.949(0.021)$ & $12.570(0.03)$ & SpT: DA + dM (McCleery et al. 2020) \\
\hline 4478524169500496000 & HD 169889 & - & 276.590 & 8.613 & $28.279(0.025)$ & $8.10(0.03)$ & SpT: G9V + WD (McCleery et al. 2020) \\
\hline 1550299304833675392 & WD $1324+458$ & - & 201.720 & 45.545 & $32.772(0.021)$ & $11.962(0.03)$ & SpT: M3V + DA (McCleery et al. 2020) \\
\hline
\end{tabular}


Table A1 - continued

\begin{tabular}{|c|c|c|c|c|c|c|c|}
\hline ptaia DR3 ID & $\begin{array}{c}\text { WD name } \\
\text { (confirmed WDs only) }\end{array}$ & $P_{\mathrm{WD}}$ & $\begin{array}{l}\text { RA } \\
\left({ }^{\circ}\right)\end{array}$ & $\begin{array}{l}\text { Dec. } \\
\left({ }^{\circ}\right)\end{array}$ & $\begin{array}{l}\varpi\left(\sigma_{\varpi}\right) \\
(\mathrm{mas})\end{array}$ & $\begin{array}{l}G\left(\sigma_{\mathrm{G}}\right) \\
(\mathrm{mag})\end{array}$ & Comment \\
\hline \multicolumn{8}{|c|}{ (E) EDR3 and DR2 catalogue entries without spectral confirmation } \\
\hline 283928743068277376 & WD J050600.41+590326.89 & 1.000 & 76.501 & 59.055 & $27.731(0.332)$ & $19.635(0.004)$ & Unobserved high $P_{\mathrm{WD}}$ DR2 candidate (table A1 of McCleery et al. 2020) \\
\hline 3346787883122375680 & WD J055602.01+135446.71 & 0.996 & 89.0100 & 13.910 & $36.531(0.084)$ & $16.928(0.002)$ & Unobserved high $P_{\mathrm{WD}}$ DR2 candidate (table A1 of McCleery et al. 2020) \\
\hline 611074413433751680 & WD J090834.39+172148.53 & 0.914 & 137.142 & 17.362 & $30.676(0.058)$ & $16.591(0.002)$ & Unobserved low $P_{\mathrm{WD}}$ DR2 candidate (table A2 of McCleery et al. 2020) \\
\hline 3982007636324256000 & WD J110143.04+172139.39 & 0.994 & 165.427 & 17.359 & $34.668(0.053)$ & $15.973(0.003)$ & Unobserved high $P_{\mathrm{WD}}$ DR2 candidate (table A1 of McCleery et al. 2020) \\
\hline 4004185576130620288 & WD J115007.08+240403.54 & 1.000 & 177.526 & 24.064 & $33.198(0.284)$ & $19.357(0.004)$ & Unobserved low $P_{\mathrm{WD}}$ DR2 candidate (table A2 of McCleery et al. 2020) \\
\hline 1688618481786030336 & WD J131830.01+735318.25 & 0.964 & 199.622 & 73.888 & $27.448(0.138)$ & $16.864(0.005)$ & Unobserved low $P_{\mathrm{WD}}$ DR2 candidate (table A2 of McCleery et al. 2020) \\
\hline 2149331587745863680 & WD J181548.96+553232.22 & 0.747 & 273.954 & 55.541 & $26.365(0.051)$ & $17.098(0.002)$ & Unobserved low $P_{\mathrm{WD}}$ DR2 candidate (table A2 of McCleery et al. 2020) \\
\hline 2127093140445053696 & WD J191936.23+452743.55 & 0.866 & 289.900 & 45.463 & $35.639(0.035)$ & $16.438(0.002)$ & Unobserved low $P_{\mathrm{WD}}$ DR2 candidate (table A2 of McCleery et al. 2020) \\
\hline
\end{tabular}

This paper has been typeset from a $\mathrm{T}_{\mathrm{E}} \mathrm{X} / \mathrm{LT} \mathrm{E} \mathrm{X}$ file prepared by the author. 